\title{
A Tractable Model of Reciprocity and Fairness
}

\author{
James C. Cox \\ Georgia State University \\ jccox@gsu.edu \\ Daniel Friedman \\ University of California, Santa Cruz \\ dan@cats.ucsc.edu \\ Steven Gjerstad \\ Purdue University \\ gjerstad@purdue.edu
}

February 14, 2006

\begin{abstract}
We introduce a parametric model of other-regarding preferences in which my emotional state determines the marginal rate of substitution between my own and others' payoffs, and thus my subsequent choices. In turn, my emotional state responds to relative status and to the kindness or unkindness of others' choices. Structural estimations of this model with six existing data sets demonstrate that other-regarding preferences depend on status, reciprocity, and perceived property rights.
\end{abstract}

We are grateful to the National Science Foundation for research support (grant numbers SES-9818561 and DUE-0226344). We are also grateful to Ken Binmore, Gary Charness, Steffen Huck, Lori Kletzer, Lisa Rutstrum, Daniel Zizzo and two anonymous referees for helpful comments. 
Before any thing, therefore, can be the complete and proper object, either of gratitude or resentment, it must possess three different qualifications. First it must be the cause of pleasure in the one case, and of pain in the other. Secondly, it must be capable of feeling these sensations. And, thirdly, it must not only have produced these sensations, but it must have produced them from design, and from a design that is approved of in the one case and disapproved of in the other.

- Adam Smith (1759, p. 181)

\section{Introduction}

Everyone knows that people care about other people. Economists have known it at least since Adam Smith, but only recently have begun to recognize the need for explicit models. Under what circumstances will I bear a personal cost to help or harm you? What is the marginal rate of substitution between my own payoff and yours? The goal of this paper is to propose a model that addresses such questions and, using some existing laboratory data, to illustrate its application.

Many things may affect how I care about you, but two general considerations stand out. One is status, or relative position: are you a member of my family, or my boss or my employee? More generally, do you have a more or less privileged position that I regard as legitimate? Such distinctions can alter my perceived obligations to you. A second concern is reciprocity. If I think you have helped me in the past or want to help me in the future, I am more likely to value your welfare. Of course, economists are familiar with folk theorem arguments that I help you now so that you will help me later and thereby increase the net present value of my payoff stream. Reciprocity in the present paper refers to something quite different, although complementary: if you are my friend, I find it pleasurable to increase your material payoff, whether or not it affects the present value of my own material payoff. Negative reciprocity is also included: if you are my foe (e.g., I think you have harmed me or want to do so), I enjoy decreasing your material payoff. Smith (1759) refers to these emotions as the "moral sentiments" of gratitude and resentment, and suggests three necessary conditions for their proper expression.

Our model formalizes the idea. In the model, status and reciprocity affect my emotional 
state, summarized in a scalar variable $\theta$, and my emotional state affects my choices. Smith's resentment corresponds to negative $\theta$, and gratitude corresponds to positive $\theta$. The model retains the conventional assumption that I choose an available alternative that maximizes my utility function, and follows recent contributions in allowing the utility function to depend on your material payoff $y$ as well as my own material payoff $m$. The simplest example is $u(m, y)=m+\theta y$. The key innovation is to model the emotional state $\theta$ as systematically affected by the reciprocity motive $r$ as well as by the status $s$.

Section 2 sets the stage by summarizing some recent related literature. Section 3 proposes specifications of the model elements $r, s$, and $\theta(r, s)$, and proposes a more general utility function that allows non-linear indifference curves. This utility function parsimoniously captures various concerns regarding other players' payoffs, e.g., a concern for efficiency as well as a greater concern for those with lower incomes.

Sections 4 through 8 apply the model to existing laboratory data. Each of these applications builds on those that precede it in a natural way. In Section 4 we estimate utility function parameters from a dictator game. The results indicate that decision makers have other-regarding preferences that can be captured by a utility function with convex indifference curves, and that dictators exhibit residual altruism. Section 5 examines a Stackelberg duopoly game. The results confirm reciprocity: the generosity of the first mover's output decision affects the second mover's emotional state and output choice. Section 6 examines mini-ultimatum games in which the alternatives available to proposers vary across games. We find again that reciprocity significantly affects responder behavior, and we also find that responders' reference payoff levels (or "property rights") depend on the alternatives open to first movers. By contrast, currently popular models of unconditional distributional preferences predict that the alternatives not chosen have no effect.

Section 7 examines the effects of status as well as reciprocity in an ultimatum game with both random and contest role assignment treatments. Section 8 briefly describes further applications. These include analysis of the earned income treatment from the dictator game in Section 4; a demonstration - based on a dictator treatment from an investment game experiment - that decision makers with a positive emotional state can exhibit a preference for efficiency; and analysis of a game with complicated choice sets, which demonstrates the 
flexibility and generality of both our utility model and our estimation procedure. Section 9 presents a concluding discussion. Technical details appear in the appendices.

\section{Recent approaches}

Economic models traditionally assume that decision-makers are exclusively motivated by material self-interest. Maximization of own material payoff predicts behavior quite well in many contexts. Examples include competitive markets, even when gains from trade go almost entirely to sellers or almost entirely to buyers (Smith and Williams, 1990); one-sided auctions with independent private values (Cox and Oaxaca, 1996); procurement contracting (Cox, Isaac, Cech, and Conn, 1996); and search (Cason and Friedman, 2003; Cox and Oaxaca, 1989, 2000; Harrison and Morgan, 1990).

Maximization of own material payoff predicts poorly in a variety of other contexts. Examples include ultimatum games (Güth, Schmittberger, and Schwarze, 1982; Slonim and Roth, 1998), dictator games (Forsythe, Horowitz, Savin, and Sefton, 1994; Andreoni and Miller, 2002), investment games (Berg, Dickhaut, and McCabe, 1995; Cox, 2004), public goods games with voluntary contributions (especially such games that allow costly opportunities for punishing free riders, e.g., Fehr and Gächter, 2000a), and experimental labor markets (e.g., Fehr, Gächter, and Kirchsteiger, 1997). Fehr and Gächter (2000b) summarize recent evidence on the economic impact of motives beyond self-interest.

The laboratory data, together with suggestive field data, have encouraged the development of models of other-regarding preferences. This literature falls into two broad classes. First there are the relative payoff (or distributional) models of Fehr and Schmidt (1999), Bolton and Ockenfels (2000), and Cox and Sadiraj (2005) and the basic ("intentions-free") model in Charness and Rabin (2002). To facilitate comparison with our specifications, we write out two-player versions of these models.

The Fehr-Schmidt model has piecewise linear indifference curves for inequality-averse preferences over my income $m$ and your income $y$. The utility function is

$$
u(m, y)= \begin{cases}m-\alpha(y-m), & \text { if } m<y \\ m-\beta(m-y), & \text { if } m \geq y\end{cases}
$$


where the marginal rate of substitution parameters satisfy $0 \leq \beta \leq \alpha$ and $\beta<1$. That is, I always like own income and the (algebraic) sign of the marginal rate of substitution between my income and yours depends on which of us has higher income. For two players, the Charness-Rabin distributional model has a similar piecewise linear form: their analysis emphasizes quasi-maximin preferences $(1>\beta>-\alpha>0)$, and can also accommodate competitive preferences $(\beta<0<\alpha)$ as well as inequality- or difference-averse preferences $(\alpha>0, \beta>0)$. The Bolton-Ockenfels two-player model also assumes that I like own income and dislike income inequality, but the utility function takes the non-linear form $u(m, y)=v(m, m /(m+y))$. They assume that the function $v$ is globally non-decreasing and concave in the first argument, strictly concave in the second argument (relative income $m /(m+y))$, and satisfies $v_{2}(m, 1 / 2)=0$ for all $m$. The Cox and Sadiraj (2005) two-player model includes nonlinear indifference curves for other-regarding preferences. The utility function has the form

$$
u(m, y)= \begin{cases}\left(m^{\alpha}+\theta y^{\alpha}\right) / \alpha, & \text { if } \alpha \in(-\infty, 0) \cup(o, 1) \\ m y^{\theta}, & \text { if } \alpha=0,\end{cases}
$$

with the parameter restriction $\theta \in[0,1)$. As in the Fehr-Schmidt model, I like own income and my marginal rate of substitution depends on whose income is higher but, in contrast to Fehr-Schmidt, I always like or am at least neutral with regard to your income and always have a decreasing marginal rate of substitution between my income and your income as my income increases.

The main alternatives so far to these distributional preference models are equilibrium models that try to capture the reciprocity motive in terms of beliefs regarding intentions. Building on the psychological games literature (e.g., Geanakoplos, Pearse and Stacchetti, 1989), Rabin (1993) develops a theory of fairness equilibria (for two player games in normal form) based on the following representation of agents' utilities. Define $a_{i}, b_{j}$, and $c_{i}$, respectively, as the strategy chosen by player $i$, the belief of player $i$ about the strategy chosen by player $j$, and the belief by player $i$ about the belief by player $j$ about the strategy chosen by player $i$. Rabin (1993, pp. 1286-7) writes the expected utility function for player $i$ as $U_{i}\left(a_{i}, b_{j}, c_{i}\right)=\pi_{i}\left(a_{i}, b_{j}\right)+\tilde{f}_{j}\left(b_{j}, c_{i}\right)\left[1+f_{i}\left(a_{i}, b_{j}\right)\right]$, where $\pi_{i}\left(a_{i}, b_{j}\right)$ is the monetary 
payoff to player $i, \tilde{f}_{j}\left(b_{j}, c_{i}\right)$ is player $i$ 's belief about how kind player $j$ is being to him, and $f_{i}\left(a_{i}, b_{j}\right)$ is how kind player $i$ is being to player $j$ (relative to a benchmark taken to be the average of the highest and lowest possible payoffs). Thus negative reciprocity $\left(\tilde{f}_{j}<0\right.$ and $\left.f_{i}<-1\right)$ as well as positive reciprocity increases utility. The model looks for equilibria in actions and beliefs about intended kindness; typically there are many such equilibria.

Dufwenberg and Kirchsteiger (2004) propose an extension to extensive form games with $N$ players, and Falk and Fischbacher (2006) propose a different extension that also covers incomplete information but uses a distributional preference utility function. Charness and Rabin (2002), in addition to their distributional model, propose an equilibrium model involving distributional preferences and beliefs about other players intentions. All the equilibrium models are complex and have many equilibria, and so seem intractable in most applications. Such problems seem unavoidable for models that assume equilibrium in higher order beliefs.

Levine (1998) improves tractability by replacing beliefs about others' intentions with estimates of others' types. In his model, players' utilities are linear in their own monetary payoff $m$ and in others' monetary payoffs $y$. For two player games, my utility is $u(m, y)=$ $m+\frac{a_{m}+\lambda a_{y}}{1+\lambda} y$, where $a_{m} \in(-1,1)$ is my type or "coefficient of altruism," $a_{y} \in(-1,1)$ is my current estimate of your type, and $\lambda \in[0,1]$ is a weight parameter. Levine demonstrates that his model is consistent with data from some ultimatum games and market experiments, and it clearly is more tractable than the previous equilibrium models.

We propose a more drastic simplification. Instead of beliefs or type estimates we use emotional states based on experience: my attitude towards your payoff depends on my state of mind, e.g., kind or vengeful, and your actual behavior systematically alters my emotional state. ${ }^{1}$ Our model is consistent with the axiomatic approaches of Sobel (2005) and Guttman (2000) but is more explicit. It is simply a preference model, not an equilibrium model, and therefore sidesteps many of the complications involving higher order beliefs. But unlike the distributional preference models discussed above, in our model an agent's distributional preferences are conditional on relative status, on the previous behavior (or, one might say, the revealed intentions) of others, and on the set of alternative actions available to others,

\footnotetext{
${ }^{1}$ Bosman and van Winden (2002) report experiments on the effects of emotions on behavior in fairness games.
} 
which often affect perceived property rights in the game.

Recent experiments compare the explanatory power of earlier models. Several experiments produce evidence contrary to the (unconditional) distributional preference models. Engelmann and Strobel (2004) report dictator experiments in which a large majority of subjects make choices that are inconsistent with unconditional inequality-averse (or differenceaverse or inequity-averse) preferences. Cox and Sadiraj (2005) report data from two- and four-agent dictator experiments in which the responses of most subjects are inconsistent, respectively, with unconditional inequality-averse and quasi-maximin preferences; they also demonstrate that the unconditional CES utility function in their paper is consistent with data from these experiments. Kagel and Wolfe (2001) find that rejection rates in the ultimatum game are essentially unaffected by unequal (high or low) contingent payments to a passive third player. Their results are contrary to both inequality aversion and to maximin preferences. Charness and Rabin (2002) evaluate two- and three-person sequential games. When the game tree is truncated, choices in dictator subgames often differ substantially from choices in the same branch of the larger game, which demonstrates that responders' choices depend on the set of alternatives from which proposers choose. In mini-ultimatum games, Falk, Fehr and Fischbacher (2003) find that rejection rates depend systematically on alternative offers not chosen by the proposers. We analyze their data in Section 6 . Brandts and Charness $(2000)$ consider $2 \times 2$ bimatrix games preceded by a cheap talk stage and followed by a costly opportunity to reward or punish the other player. They find that deception in the cheap talk stage significantly increases the punishment rate, and that some subjects reward favorable sender behavior. Blount (1995) finds that responders in her ultimatum games accept lower offers more often when they are randomly generated than when they are chosen by human subjects. Offerman (2002) has similar results: intentional helpful (hurtful) actions are rewarded (punished) more frequently than identical but randomly generated actions. A number of more recent papers arrive at similar conclusions. There are some empirical studies that seem more favorable to unconditional distributional preferences than to reciprocal preferences, including Bolton, Katok and Zwick (1998) and Bolton, Brandts and Ockenfels (1998), but the preponderance of evidence supports the empirical significance of reciprocity and context dependence as elements of distributional preferences. 
Cox $(2002,2004)$ uses a triadic experimental design to discriminate between actions motivated by unconditional distributional preferences and actions motivated by reciprocity considerations, in the context of the Berg, Dickhaut, and McCabe (1995) investment game. Using dictator game treatments as controls, the experiments support the conclusion that behavior is significantly motivated by altruism as well as by trust and positive reciprocity. Cox, Sadiraj, and Sadiraj (2004) apply the triadic design to the moonlighting game introduced by Abbink, Irlenbusch, and Renner (2000), and report that altruism and positive reciprocity (but not negative reciprocity) are significant motives. Cox and Deck (2005) apply the triadic design to eleven experimental treatments involving 692 subjects and various twoperson games. They find significant positive reciprocity in the trust (or mini-investment) game when it is run with a single-blind protocol but not when it is run with a double-blind protocol. They do not find significant negative reciprocity in the "punishment" game (i.e., the $5 / 5$ mini-ultimatum game) when it is run with a double blind protocol in a triadic design.

In summary, the laboratory evidence confirms that people do care about others' payoffs as well as their own. The marginal rate of substitution (between my payoff and yours) is not constant, however, and may be affected by reciprocity as well as distributional and status considerations. There is need for a tractable model that can be successfully applied to data from games that differentially reflect subjects' concerns for reciprocity, distribution, and status.

\section{Model specifications}

This section presents a model of preferences that incorporates objectively defined variables $r$ and $s$ capturing the effects of reciprocity and status considerations on behavior. For pedagogical and comparative purposes, the presentation here considers only two player extensive form games of perfect information with first mover $F$ receiving material payoff $y$, and second mover $S$ receiving material payoff $m$. The model shows how the emotional state of $S$ defines the marginal rate of substitution $(M R S)$ between own payoff $m$ and other's payoff $y$, and how the emotional state responds to the values of $r$ and $s$ that arise from $F$ 's 
prior choice and relative status.

Due to their importance in existing literature, concerns regarding distribution or relative payoff are separated from other aspects of status, and are captured in the convexity of indifference curves in the $(m, y)$ payoff space. To see this clearly, suppose for the moment that both payoffs are positive and that the second mover has kind preferences (i.e., increasing in both own and other's payoff). Indifference curves then have the usual negative slope. If preferences are convex, the $M R S$ increases as one moves along any indifference curve in the direction of increasing $y / m$ ratio. But $y / m$ is a natural way to specify relative payoff. The $M R S$ is independent of $y / m$ when indifference curves are linear, and greater sensitivity to $y / m$ takes the form of more convex preferences.

With homothetic preferences, all indifference curves have the same slope where they cross any given ray, $y / m=$ constant; in this case relative payoff dependence is well defined. Fortunately the convenient and well-known constant elasticity of substitution (CES) utility function represents homothetic preferences. In its most familiar form, the CES utility function is $u(m, y)=\left(m^{\alpha}+\theta y^{\alpha}\right)^{1 / \alpha}$; see also Andreoni and Miller (2002). ${ }^{2}$

We modify this function slightly. The exponent $1 / \alpha$ is problematic when it applies to a negative expression, which arises when $\theta$ is sufficiently negative. Of course, the outside exponent doesn't affect the convexity of the indifference curves, but its sign affects their ordering. The ordering is preserved and the negativity issue is finessed by using the factor $1 / \alpha$. Hence the preference model is defined for elasticity of substitution (or convexity) parameter $\alpha \in(-\infty, 1]$ by

$$
u(m, y)= \begin{cases}\left(m^{\alpha}+\theta y^{\alpha}\right) / \alpha, & \alpha \in(-\infty, 0) \cup(0,1] ; \\ m y^{\theta}, & \alpha=0 .\end{cases}
$$

With these preferences we have $M R S=\frac{\partial u / \partial m}{\partial u / \partial y}=\theta^{-1}\left(\frac{y}{m}\right)^{1-\alpha}$. Hence the emotional state $\theta$ is the willingness to pay own for other's payoff $(W T P=1 / M R S)$ at an allocation on the equal payoff line $m=y$. Preferences are linear (and $M R S$ is constant) if $\alpha=1$, and preferences are strictly convex (and $M R S$ strictly increases in relative payoff $y / m$ along

\footnotetext{
${ }^{2}$ Fisman, Kariv and Markovits (2005) recently considered CES and more general convex preferences to explain choices in dictator games.
} 
indifference curves) if and only if $\alpha<1$. Appendix A.1 shows that indifference curves for $\alpha \neq 0$ converge pointwise as $\alpha \rightarrow 0$ to indifference curves of the Cobb-Douglas preferences $u(m, y)=m y^{\theta}$. A standard textbook argument shows that for positive values of $\theta$, as $\alpha \rightarrow-\infty$, the indifference curves for $\theta>0$ converge to Leontief indifference curves with corners on the ray $y / m=\theta^{-1}$.

The emotional state $\theta$ is a function of the reciprocity and status variables $r$ and $s$. A natural specification for the reciprocity variable is $r(x)=m(x)-m_{0}$, where $m(x)$ is the maximum payoff the second mover can guarantee himself given the first mover's choice $x$, and $m_{0}$ is $m\left(x_{0}\right)$ when $x_{0}$ is neutral in some appropriate sense. ${ }^{3}$ Sometimes it is convenient to normalize $r(x)$ so that it lies in $[-1,1]$. Let $m_{g}=\max _{x} m(x)$ and $m_{b}=\min _{x} m(x)$. The normalized version is $r(x)=\left(m(x)-m_{0}\right) /\left(m_{g}-m_{b}\right)$, when $m_{g}>m_{b}$, and $r=0$ otherwise.

The variable $s$ represents relative status (other than relative payoff, which is already accounted for). It refers to a generally recognized asymmetry in players' claims or obligations, e.g., due to differences in age, sex, job title, or effort. In the current paper we examine only shifts in payoff entitlements when an advantageous role is earned rather than awarded randomly. In sections 7 and 8 below, we code status as a real number for each player, e.g., $s_{F}$ and $s_{S}$ respectively for the first and second player, and code relative status as the difference, $s=s_{F}-s_{S}$. For example, under some social norms the first mover's status and hence $s$ would increase if she had to earn the right to be the first mover or earn her endowment. These examples are analyzed in Sections 7 and 8 .

In estimating the model, we impose two assumptions.

A.1 Individuals choose so as to maximize a utility function of the form in equation (1).

A.2 The emotional state function $\theta=\theta(r, s)$ is identical across individuals except for a mean zero idiosyncratic term $\epsilon$. Player $i$ 's emotional state is then written $\theta_{i}=\theta(r, s)+\epsilon_{i}$.

\footnotetext{
${ }^{3}$ Konow (2001) elaborates an objective theory of $m_{0}$ as a function of the agent's relative actual effort levels ("accountability"), the efficient effort levels, the agents' basic material needs, and the context. Konow (2000) extends (part of) this theory to allow for self-serving subjective distortions of the objective $m_{0}$, and confronts evidence from dictator games. (In our framework, this game entails a strategic dummy first mover.) Konow (2003) surveys relevant moral philosophy and evidence. Gächter and Riedl (2003) offer a general discussion and demonstrate the impact of $m_{0}$ (which they call moral property rights or entitlements) in new laboratory data. We take a different approach and estimate $m_{0}$ from the data.
} 


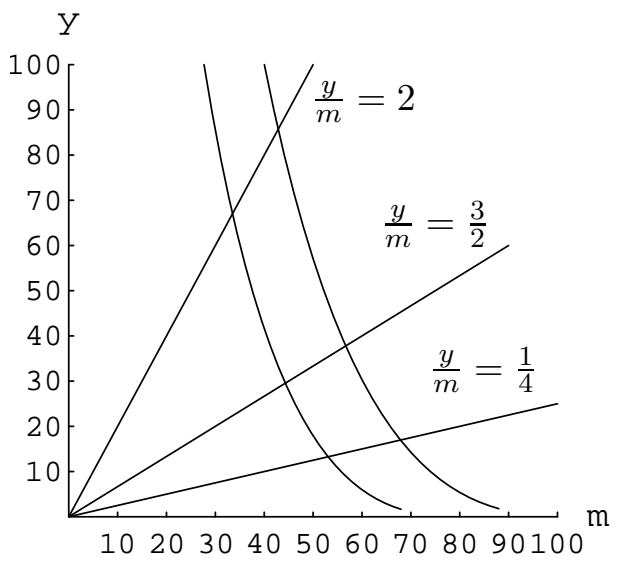

(a) Indifference curves for $\theta=0.3$.

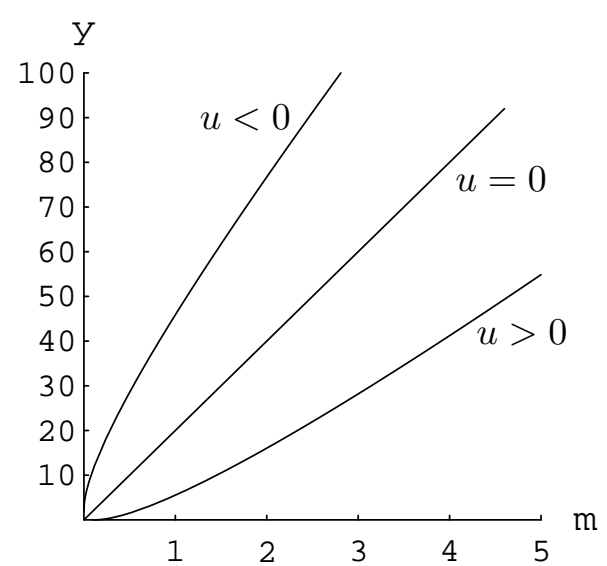

(b) Indifference curves for $\theta=-0.3$.

Figure 1: Indifference curves for the utility function $u(m, y)=2.5\left(m^{0.4}+\theta y^{0.4}\right)$.

In the applications that include reciprocity or status we test the following hypothesis.

H.1 The emotional state function $\theta(r, s)$ increases in $r$ and $s$.

Several comments are in order before proceeding. Assumption A.1 imports the CES utility function from its usual home territory (preferences over bundles of private consumption goods) and applies it to social choice territory (preferences over own and others' material payoffs). When the emotional state $\theta$ is positive and the convexity parameter is strictly between its extreme values $\alpha=-\infty$ and $\alpha=1$, assumption A.1 implies the usual smooth, downward-sloping, convex indifference curves, as in figure 1 (a). An implication of downward-sloping curves is that a person will give up some of her own payoff when that would sufficiently increase the payoff of another. Thus an individual with positive $\theta$ has a taste for efficiency, in that she will often choose to increase the sum of the two payoffs. When the optimal choice is in a neighborhood of the ray $y / m=\theta^{\frac{1}{1-\alpha}}$, it maximizes the payoff sum. Also, an implication of strict convexity of the utility function is that the individual's willingness to pay increases as the other's relative income decreases. In this sense she has maximin-like preferences. Such preferences are quite literal in the $n$-player version of the model described in Section 9: a player's willingness to pay is highest for other player who is the least well off.

Assumption A.1 implies that utility strictly increases in own payoff $m$. Whether utility increases in other's payoff $y$ depends on the sign of $\theta$. Sometimes it is useful to decompose 
$\theta(r, s)$ as $\theta(r, s)=\theta_{0}+\tilde{\theta}(r, s)$, where $\tilde{\theta}(0,0)$ is zero, and $\theta_{0}$ is the residual benevolence or malevolence. Thus the typical individual will have standard selfish preferences when

$\theta(r, s)=0$, i.e., $\tilde{\theta}(r, s)=-\theta_{0}$. Of course, assumption A.2 then implies that $\theta_{i}<0$ for some individuals at this point, while $\theta_{i}>0$ for other individuals.

It might seem natural to assume that the individual idiosyncracies in assumption A.2 have the normal (or Gaussian) distribution, but some of our data suggest otherwise. For example, the normal distribution implies that no matter how kindly the first mover behaves, an occasional second mover will have negative $\theta$, but such churlish behavior is non-existent in some of our data. Therefore our estimation techniques allow for the possibility of either platykurtotic (thin-tailed) error distributions - i.e., distributions between normal and uniform - or leptokurtotic (fat-tailed) error distributions.

We accommodate these error term characteristics with the error function (or exponential power) distribution with density

$$
f(z ; b, c)=\frac{e^{-0.5|z / b|^{2 / c}}}{b 2^{c / 2+1} \Gamma(c / 2+1)} .
$$

For $c=1$, this distribution reduces to the normal distribution with mean zero and variance $b^{2}$. As $c \rightarrow 0$, this distribution tends to a uniform density on $[-b, b]$; see Evans, Hastings, and Peacock (2000). Our estimation strategy lets the data select the error distribution (from among this class) that has the best fit rather than imposing one distribution, such as the normal, a priori.

Finally, note that assumption A.2 and hypothesis H.1 together suggest that most individuals will have negative $\theta$ when $r$ and/or $s$ are sufficiently negative. A person with negative $\theta$ is willing to pay to reduce another's payoff. That is, $y$ is a "bad" rather than a "good," and the indifference curves slope upward. CES preferences then have one straight line indifference curve, the ray $y / m=|\theta|^{-1 / \alpha}$ corresponding to $u=0$, and the slopes of other indifference curves converge towards the slope of this ray as in figure 1 (b).

\section{Altruism in dictator games}

In our first application, we examine choices in a dictator game. Although this game has an especially simple structure, it allows us to demonstrate cleanly that the utility function 
in equation (1) together with the idiosyncratic error terms in equation (2) explain choices well. Since the baseline dictator sessions include neither reciprocity nor status, the analysis spotlights the role of non-reciprocal altruism or benevolence $\theta_{0}$. In Section 8 we examine the role of status arising from an earned endowment treatment for the dictator game.

In the baseline dictator games reported by Cherry, Frykblom, and Shogren (2002), roles are assigned randomly and subjects assigned to the dictator role are provided with an endowment of either $\omega=\$ 10$ or $\omega=\$ 40$ which they split between themselves and the other player. From assumptions A.1 and A.2 the dictator's utility in this game is $u(x)=\alpha^{-1}\left((\omega-x)^{\alpha}+\left(\theta_{0}+\epsilon\right) x^{\alpha}\right)$, where $x$ is the amount offered by the dictator to the other player. For $\alpha$ positive $^{4}$, the utility maximization condition is

$$
\max _{x} u\left(x ; \alpha, \theta_{0}, \epsilon\right)=\max _{x}\left((\omega-x)^{\alpha}+\left(\theta_{0}+\epsilon\right) x^{\alpha}\right) / \alpha .
$$

The estimation strategy begins from the observation that, for given values of $\alpha$ and $\theta_{0}$, the probability that $x$ is the solution to equation (3) is the probability that $\epsilon$ falls within a particular interval $\left(\epsilon_{x-1}, \epsilon_{x}\right)$. As explained in appendix A.2, the threshold values $\epsilon_{x}$ (where the optimal actions switch) are determined by a well behaved function of the parameters $\alpha$ and $\theta_{0}$, once the distribution is specified for the idiosyncratic error $\epsilon$. From the error term distribution specification in equation (2) and the utility maximization condition in equation (3), we can write the probability of choice $x$ in the $\omega$-game in terms of the parameters as $\operatorname{Pr}_{\omega}\left[x \mid \alpha, \theta_{0}, b, c\right]$.

The Cherry, Frykblom, and Shogren baseline treatment data consist of twenty-six dictator choices made with $\omega=\$ 10$ and twenty-six choices made with $\omega=\$ 40$. Allocations to the dictator (denoted $m$ ) and to the other player (denoted $y$ ) are shown in figure 2 . Data and choice probabilities are used to write down the log likelihood function in terms of model parameters. Choice frequencies in the $\omega=\$ 10$ game were $g_{10}=(6,3,4,4,3,6)$ for $x \in\{0,1,2, \ldots, 5\}$. (No offer to the other above $x=5$ was observed in this game.) Choice frequencies in the $\omega=\$ 40$ game were $g_{40}$, where $g_{40}$ is the distribution shown in the graph on the right side of figure 2. The log likelihood function is

$$
\ln L\left(\alpha, \theta_{0}, b, c\right)=\sum_{x=0}^{5} g_{10, x} \ln P r_{10}\left[x \mid \alpha, \theta_{0}, b, c\right]+\sum_{x=0}^{20} g_{40, x} \ln \operatorname{Pr}_{40}\left[x \mid \alpha, \theta_{0}, b, c\right]
$$

\footnotetext{
${ }^{4}$ For $\alpha<0$, utility is $-\infty$ at $x=0$. Since offers of $x=0$ occur in the data set, we must have $\alpha \geq 0$.
} 

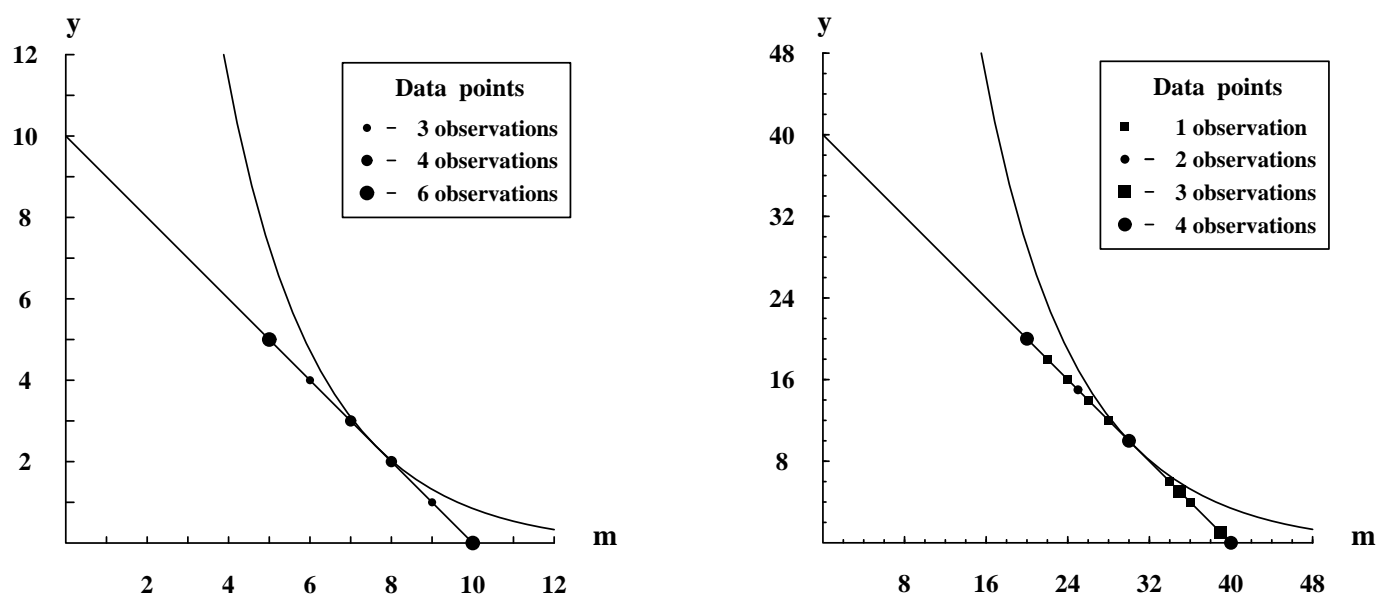

Figure 2: Data and indifference curves for estimated utility function from dictator games.

Parameter estimates - obtained by maximizing the log likelihood - and standard errors are shown in table $1 .^{5}$ We also test the hypothesis that $\theta_{0}=0$ and find that the $\chi^{2}$ test statistic is $\chi^{2}(1)=22.598$, which has $p$-value less than $p=0.0001$.

\begin{tabular}{ccc} 
Parameter & Estimate & SE \\
\hline$\alpha$ & 0.255 & 0.187 \\
$\theta_{0}$ & 0.417 & 0.064 \\
$b$ & 0.621 & 0.066 \\
$c$ & 0 & \\
\hline
\end{tabular}

Table 1: Parameter estimates from dictator game

The two graphs in figure 2 show the indifference curves from the estimated utility function (with individual-subject idiosyncratic error term set equal to its mean value of 0 ; see assumption A.2). In the $\omega=\$ 10$ dictator game in the left graph, sufficiently positive idiosyncratic terms $\epsilon_{i}$ lead to offers of 3,4 , or 5 in our utility and choice model, while sufficiently negative values of $\epsilon_{i}$ lead to offers of 0 or 1 . In the $\omega=\$ 40$ game, offers above (or below) $\$ 10$ only occur when $\epsilon_{i}$ is positive (or negative).

This application of the model highlights unconditional altruism in the dictator game data. More importantly, it demonstrates how to incorporate individual-subject differences

\footnotetext{
${ }^{5}$ Since the parameter estimate for $c$ lies on the boundary - that is, in the set of uniform distributions - standard errors are calculated from the asymptotic covariance matrix for the model restricted to uniform distributions on $[-b, b]$. Hence no standard error for $c$ is included for this application, or in later applications with the same estimate of $c$.
} 
in a tractable and parsimonious way. The application also provides a helpful benchmark for the status effect presented in Section 8, and for reciprocity in the next application.

\section{$5 \quad$ Reciprocity in Stackelberg duopoly games}

Like dictators, second movers in a Stackelberg game choose joint payoffs from a range of possible choices, but unlike dictators their utility may respond to first mover behavior. Huck, Müller, and Normann (2001) present an experiment in which randomly matched pairs of subjects play a Stackelberg duopoly game. The first mover $(F)$ chooses an output level $x \in\{3,4,5, \ldots, 15\}$. The second mover $(S)$ observes $x$ and chooses an output level $q \in\{3,4,5, \ldots, 15\}$. The price is $p=30-x-q$; both players have constant marginal cost 6 and no fixed cost, so the profit margin for each player is $M=24-x-q$. Payoffs therefore are $m=M q$ and $y=M x$ or $m(x, q)=(24-x-q) q$ and $y(x, q)=(24-x-q) x$.

Given $F$ 's choice $x$, the second mover's choice set is the locus in $(m, y)$ space traced out by varying $q$ from 3 to 15 . As illustrated in figure 3 , it is a parabolic arc that opens toward the $y$-axis whose vertex $(m, y)=\left(\frac{1}{4}(24-x)^{2}, \frac{1}{2}(24-x) x\right)$ corresponds to $q=\frac{1}{2}(24-x)$. In figure $3, F$ 's choice is $x=4 ; S$ 's choice $q=3$ produces payoff vector $(m, y)=(51,68)$ while $q=10$ produces the vertex payoff vector $(m, y)=(100,40)$. With $x=4$, choices $q<10$ reduce $m$ but increase $y$, while choices $q>10$ reduce both $m$ and $y$.

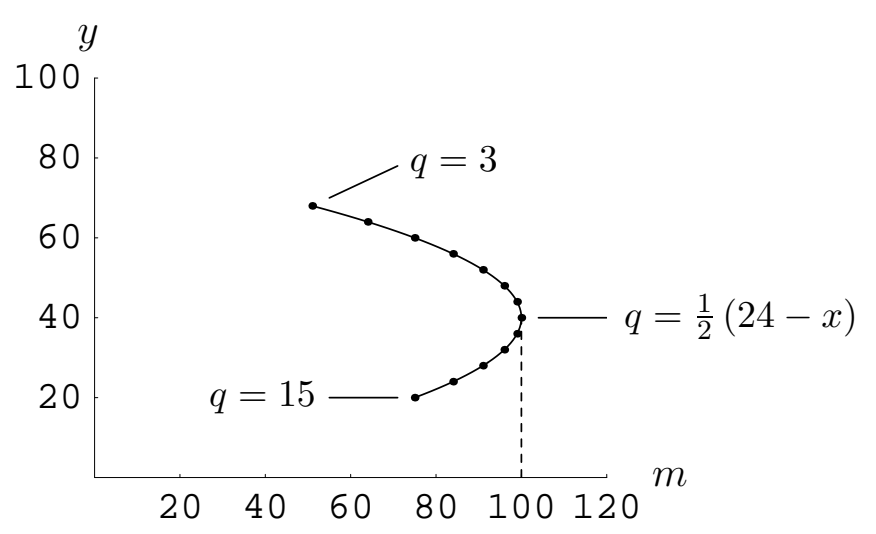

Figure 3: Feasible joint profits when first mover output is $x=4$.

In the standard textbook analysis of this game, $S$ responds to $F$ 's output $x$ by choosing the selfish best reply output $q=\frac{1}{2}(24-x)$ and obtains the $m$-maximizing (vertex) payoff 
$m(x)=\frac{1}{4}(24-x)^{2}$. Therefore $F$ chooses $x=12$ to maximize his component $y(x)=$ $\frac{1}{2}(24-x) x$ at the vertex payoff. Thus the classic Stackelberg equilibrium is $x=12$ and $q=6$, with $p=12$ and $M=6$, yielding payoffs $(m, y)=(36,72)$. In the symmetric, simultaneous move Cournot game, the classic equilibrium choices are $(x, q)=(8,8)$ so that $p=14, M=8$, and $(m, y)=(64,64)$.

The Huck, Müller, and Normann (henceforth HMN) data we analyze consist of all Stackelberg games with randomly matched players: twenty-two first and second movers each participating in ten Stackelberg games produces 220 choice pairs $\left(x_{i}, q_{i}\right)$. Output pairs are depicted in figure 4. Although the two most frequent outcomes correspond to Cournot and Stackelberg equilibrium outputs, a large fraction (125 of 220) of second mover output choices lie strictly above the standard selfish best response line $q=\frac{1}{2}(24-x)$ shown in the figure, while only 21 lie strictly below it. Moreover, the bias towards overproduction becomes more pronounced as the first mover output $x$ increases. Another striking fact is that the data are heteroscedastic: the dispersion of second mover choices increases with first mover output $x$.

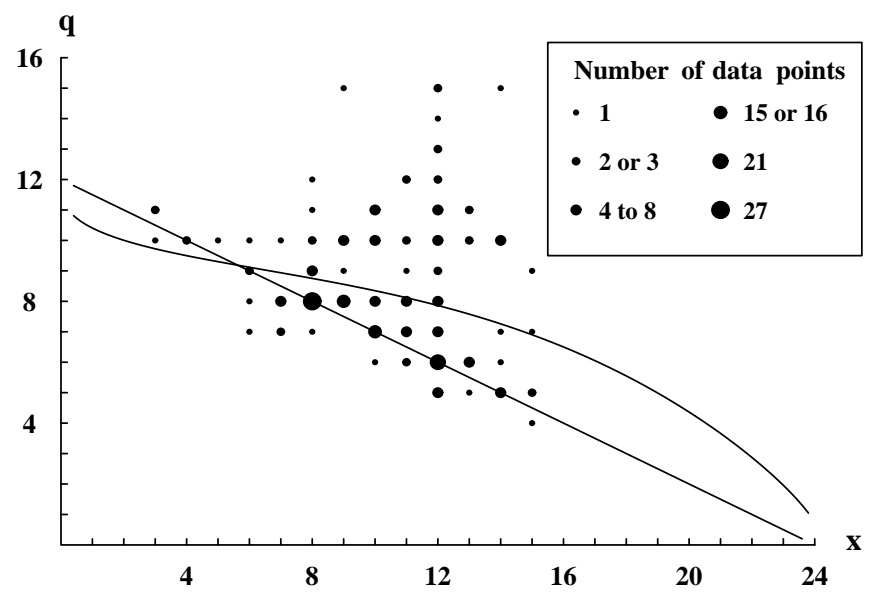

Figure 4: Choice pairs $(x, q)$ and estimated best response function.

These second mover choice patterns arise naturally from our emotional state dependent utility function. The intuition is that $F$ is greedier when he chooses a larger $x$, which pushes the reciprocity variable $r(x)$ towards more negative values. Hence $S$ has a more negative emotional state $\theta$, and therefore chooses output above the selfish best reply to reduce $F$ 's payoff $y$. The smooth curve in figure 4 indicates the best response of a second mover with such a concern for reciprocity. 
Panel (a) of figure 5 redraws $S$ 's choice set from figure 3 given $x=4$, and also includes a tangent indifference curve for positive $\theta$. Here $S$ chooses $q$ slightly below the selfish best reply $q=10$, reducing his payoff a bit below $m(4,10)=100$ to $m(4,9)=99$ while boosting $F$ 's payoff noticeably above $y(4,10)=40$ to $y(4,9)=44$. Panel (b) shows $S$ 's choice set given $F$ 's much less generous choice $x=12$, and the tangent indifference curve for a negative $\theta$. Due again to the parabolic choice set, by increasing $q$ above the selfish best response, $S$ obtains a first-order decrease in $F$ 's payoff from $y(12,6)=72$ to $y(12,7)=60$ while sacrificing only a second-order amount of his own payoff from $m(12,6)=36$ to $m(12,7)=35$. This illustrates the basic point that $S$ is more inclined to overproduce (relative to selfish best response) when the output of $x$ by $F$ is larger.

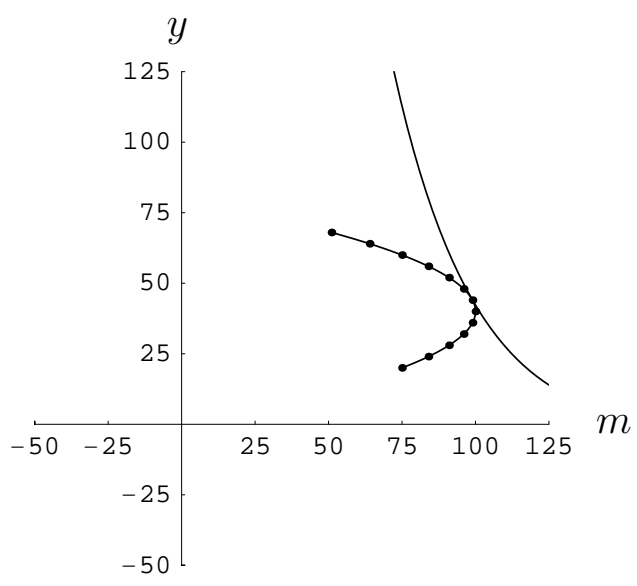

(a) Indifference curve for $x=4$.

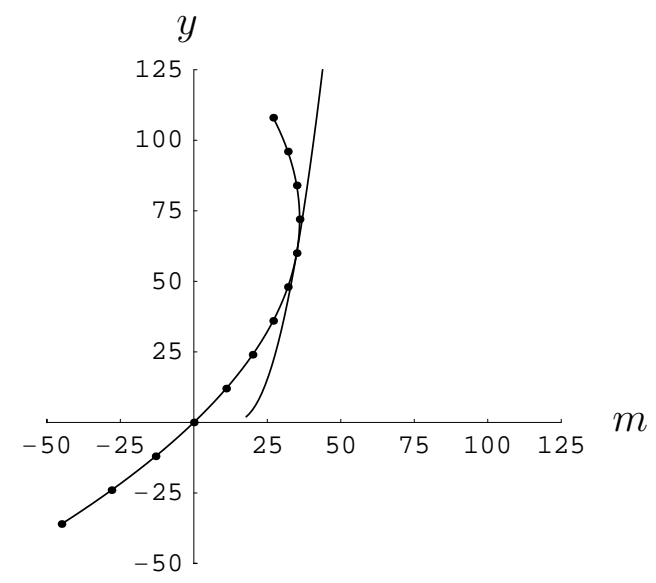

(b) Indifference curve for $x=12$.

Figure 5: Indifference curves for utility function estimated from HMN data.

The model provides a natural explanation for heteroscedasticity in the choices of $q$ even though, by assumption A.2, $S$ 's underlying individual variability $\epsilon$ is independent of first mover choice $x$. The explanation starts by noting that in panel (a) of figure 5 , the choice set and the indifference curves for $x=4$ have very different curvatures. Hence the tangency point doesn't move much as one changes $\epsilon$. By contrast, in panel (b) for $x=12$, the curvature of the choice set is much closer to the curvature of the indifference curve, so the same change in $\epsilon$ has a much larger impact on the tangency point. Thus the model implies that second mover choices $q$ will be more dispersed when the first mover choice $x$ is larger.

The data analysis begins by working out reciprocity and status. In this application, the 
status variable $s$ is constant, so we set $s=0$. Assumption A.2 and a first-order Taylor series expansion allow us to write the emotional state function as $\theta_{i}=\theta_{0}+a r(x)+\epsilon_{i}$. With $r(x)=\left(m(x)-\tilde{m}_{0}\right)$ this can be written as

$$
\begin{aligned}
\theta_{i} & =\theta_{0}+a\left(m(x)-\tilde{m}_{0}\right)+\epsilon_{i} \\
& =a\left(m(x)-\left(\tilde{m}_{0}-\theta_{0} / a\right)\right)+\epsilon_{i} .
\end{aligned}
$$

The residual benevolence term $\theta_{0}$ cannot be identified separately from the property rights $m_{0}$ in this experiment. The definition $m_{0} \equiv \tilde{m}_{0}-\theta_{0} / a$ absorbs $\theta_{0}$ into $m_{0}$.

For this application we normalize reciprocity as $r(x)=\left(m(x)-m_{0}\right) /\left(m_{g}-m_{b}\right)$, where $m_{g}-m_{b}=m(3)-m(15)=90$ so $r(x)=\left((12-0.5 x)^{2}-\left(12-0.5 x_{0}\right)^{2}\right) / 90$ for a suitably defined neutral first mover choice $x_{0}$. Then

$$
\theta_{i}=a\left((12-0.5 x)^{2}-\left(12-0.5 x_{0}\right)^{2}\right) / 90+\epsilon_{i} .
$$

In interpreting the estimate of the neutral first mover choice $x_{0}$, one should remember that it incorporates residual benevolence as well as property rights. This convention does not affect our test of assumption H.1, since absorption of $\theta_{0}$ into $m_{0}$ does not affect $a$.

Now write the second mover's utility function in terms of the players' choices by substituting the payoff functions $m(x, q)$ and $y(x, q)$ into equation (1) to obtain

$$
u_{i}(x, q)= \begin{cases}\frac{1}{\alpha}(24-x-q)^{\alpha}\left(q^{\alpha}+\theta_{i} x^{\alpha}\right), & \alpha \in(-\infty, 0) \cup(0,1], \\ (24-x-q)^{1+\theta_{i}} q x^{\theta_{i}}, & \alpha=0 .\end{cases}
$$

Set the derivative of (5) with respect to $q$ equal to 0 , and simplify to obtain the first order condition $(24-x-2 q) q^{\alpha-1}-\theta_{i} x^{\alpha}=0$. Although this first-order condition is valid for all $\alpha \leq 1$, it can be solved for $q=q^{*}(x ; \theta, \alpha)$ in closed form only in special cases. Appendix A.3 demonstrates that a unique maximizer for equation (5) exists for every parameter vector $(\theta, \alpha) \in(-\infty, \infty) \times(-\infty, 1]$, so the best response $q^{*}(x ; \theta, \alpha)$ is well defined. Appendix A.4 describes the algorithm used to determine $q^{*}(x ; \theta, \alpha)$.

Using equation (4), expand $q^{*}(x ; \alpha, \theta)$ as $q^{*}\left(x ; \alpha, a, x_{0}\right)$. As in the previous application, we assume that $\epsilon$ has an error function distribution, with the density in equation (2). The estimation procedure finds the convexity parameter $\alpha$, the reciprocity sensitivity $a$, the reference output $x_{0}$, and the error distribution parameters $b$ and $c$ that maximize the log 
likelihood function $\ln L\left(\alpha, a, x_{0}, b, c ; x, q\right)=\sum_{i=1}^{220} \ln \operatorname{Pr}\left[q=q_{i} \mid x_{i}, \alpha, a, x_{0}, b, c\right]$ for the 220 observed choice pairs $\left(x_{i}, q_{i}\right){ }^{6}$ The estimation procedure is described in appendix A.5.

\begin{tabular}{ccc} 
Parameter & Estimate & $\mathrm{SE}$ \\
\hline$\alpha$ & 0.285 & 0.129 \\
$a$ & 0.789 & 0.101 \\
$x_{0}$ & 5.669 & 0.564 \\
$b$ & 0.494 & 0.044 \\
$c$ & 0.549 & 0.116 \\
\hline
\end{tabular}

Table 2: Parameter estimates and standard errors for the Stackelberg game

Table 2 shows parameter estimates and standard errors. The estimated best-response function is shown in figure 4; figure 5 shows indifference curves from the estimated utility function that correspond in one case to $x=4$ and in the other to $x=12$.

Parameter estimates allow us to test hypothesis H.1, that the emotional state $\theta$ increases in reciprocity $r$. The null hypothesis is that $a=0$, i.e., that second movers may respond to distributional concerns but they do not respond to reciprocity concerns. For the constrained model, we write $\theta=\theta_{0}+a\left(m(x)-\tilde{m}_{0}\right)$ and set $a=0$. The likelihood ratio test statistic for this constraint is $\chi^{2}(1)=34.784$, firmly rejecting the null hypothesis at a $p$-value of less than $10^{-7}$, in favor of hypothesis H.1. Thus we find strong evidence of reciprocal behavior by Stackelberg followers in the HMN data. The estimate of the property right (or neutral first mover output choice) $\hat{x}_{0}=5.669$ is well below the Stackelberg outcome, and even somewhat lower than the Cournot outcome. Thus reciprocity is primarily negative, since first mover outputs above the low reference level $\hat{x}_{0}=5.669$ result in second mover output above the standard best response.

The very positive $\hat{a}$ estimate is strong but indirect evidence that the HMN data reflect reciprocity and not just inequality aversion. A piece of direct evidence is that 11 of 38 responses to $x=8$ were $q>8$ and one was $q<8 .{ }^{7}$ These responses are consistent with our

\footnotetext{
${ }^{6}$ Six of 220 output pairs result in negative payoffs to both players. Since our utility function is defined only for nonnegative payoffs, we truncate these six observations $q_{i}$ to $q_{i}^{\prime}=24-x_{i}$ implying zero payoffs.

${ }^{7}$ In this game, second mover output $q=8$ is the only response consistent with inequality aversion when the first mover chooses output $x=8$. For $x=8$, the line $y=m$ passes through the vertex payoffs, so that for any inequality averse utility function, the vertex payoff maximizes utility.
} 
model, given the property right estimate, but are inconsistent with inequality aversion (or selfish optimization). ${ }^{8}$

This application demonstrates that reciprocity (and especially negative reciprocity) appears to be a very significant motive in Stackelberg duopoly games. The model also captures individual-subject differences in reciprocity in a tractable and parsimonious way.

\section{Reciprocity and property rights in mini-ultimatum games}

The next application, mini-ultimatum games (Bolton and Zwick, 1995; Gale, Binmore, and Samuelson, 1995) ${ }^{9}$ also demonstrates the importance of reciprocity, and provides a unique insight into the second mover's perception of his property rights. In mini-ultimatum games, the first mover $F$ (the "proposer") offers one of two possible payoff vectors, and the second mover $S$ (the "responder") either accepts the offer, which then becomes the actual payoff vector, or else refuses, in which case the payoff is $(m, y)=(0,0)$.

Figure 6 illustrates the game trees employed by Falk, Fehr, and Fischbacher (2003). The top number at each terminal node in figure 6 is $F$ 's payoff $y$ and the bottom number is $S$ 's payoff $m$. In the $5 / 5$ game, for example, if $F$ chooses left ( $x=$ "Take") then $S$ chooses between payoff vectors $(m(x), y(x))=(2,8)$ and $(m, y)=(0,0)$; if $F$ chooses right $(x=$ "Share") then $S$ chooses between $(m(x), y(x))=(5,5)$ and $(m, y)=(0,0)$. Falk, Fehr, and Fischbacher (henceforth denoted FFF) employ the strategy method for second mover responses, so the forty-five pairs of first and second movers generate forty-five observations from each decision node in each game tree. Figure 6 lists (in parentheses) the observed frequencies for all choices.

The FFF data clearly contradict the traditional assumption of self-interested preferences, which implies that $S$ will never choose a zero payoff when she can obtain a positive payoff

\footnotetext{
8 An anonymous referee points out that, more generally, in our model equal payoffs can be a "benchmark for reciprocity. This offers a potential explanation to the puzzling result that the inequality aversion models appear to do very well in games where reciprocity can be an issue but appear to do much worse in games where reciprocity is irrelevant." The inconsistency of much of the Stackelberg data with inequality aversion indicates that inequality aversion models may not always perform well even in games that involve reciprocity.

${ }^{9}$ Binmore condemns the term mini-ultimatum game or MUG, which we perpetuate, and favors ultimatum mini-game. As a compromise, we urge readers to parse MUG as mini-[ultimatum game].
} 

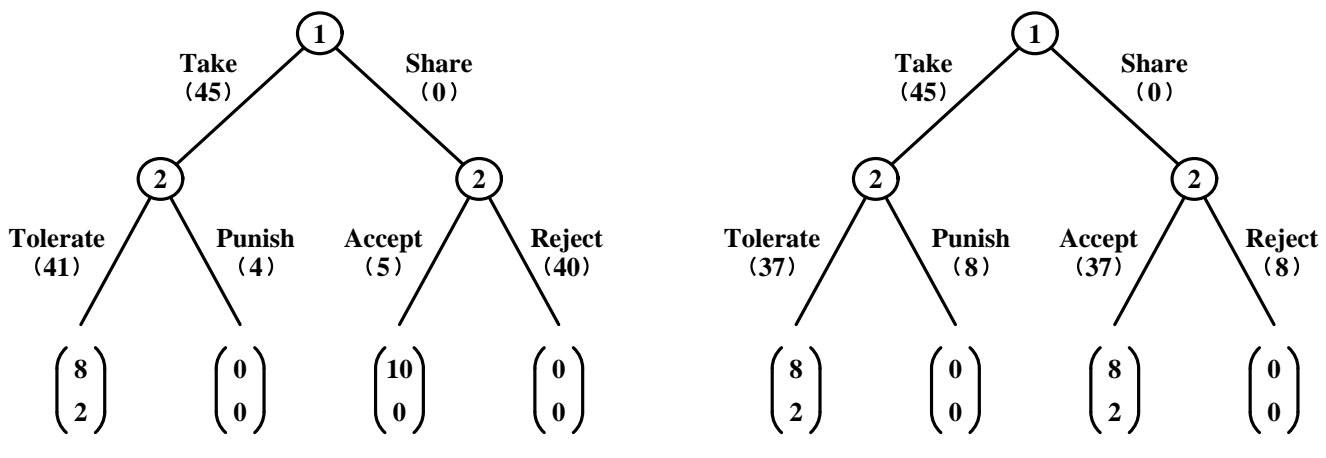

10 - 0 Game
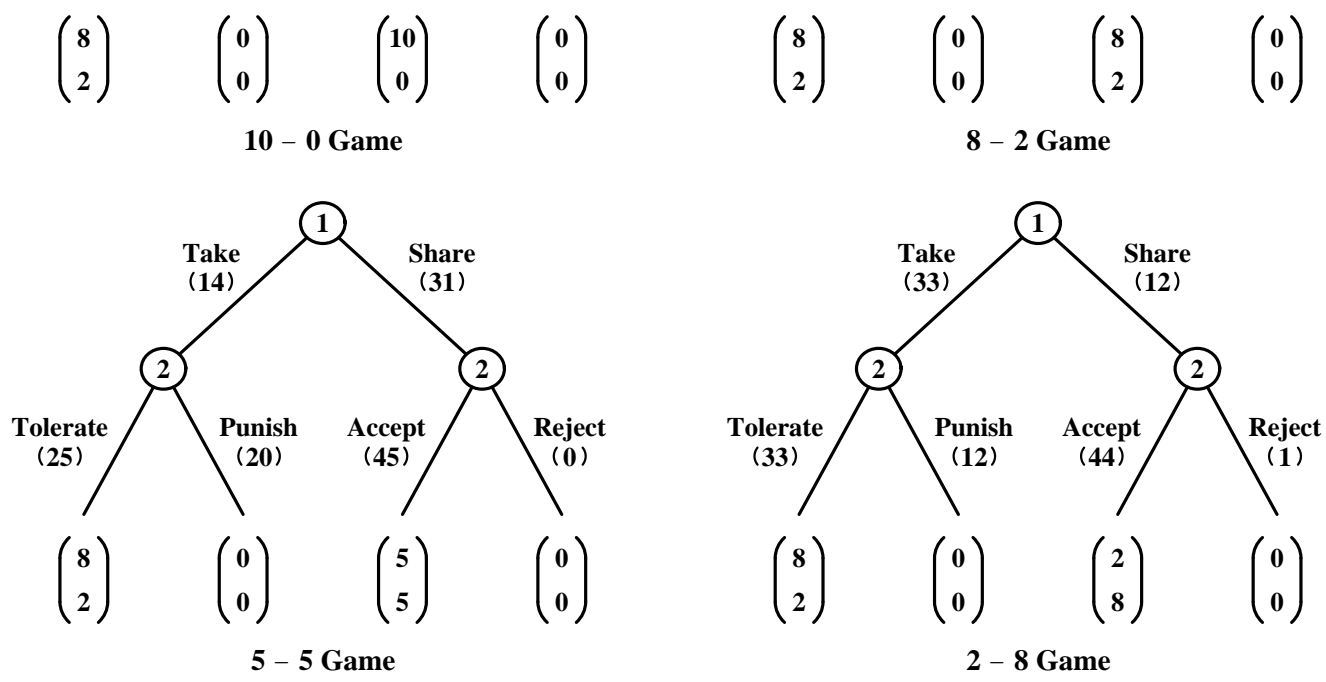

Figure 6: Extensive forms of mini-ultimatum games and action frequencies.

(e.g., $S$ will never choose to Punish). The data also contradict the distributional models reviewed earlier, which imply that $S$ cares only about the final allocation, not how it came about. In the FFF data, the Punish rate of the $(m, y)=(2,8)$ offer is almost $50 \%$ in the $5 / 5$ game where $F$ could have offered an even split, while the Punish rate of the same offer is less than $10 \%$ in the $10 / 0$ game where $F$ 's alternative is to keep the entire pie to himself.

These features of the data are natural consequences in our model of the reciprocity variable $r(x)$ in the emotional state (or WTP) function $\theta$ for the second mover. A more generous offer by $F$ increases $r(x)$, which in turn increases the value of $\theta$, thus reducing the probability that $S$ will choose to Punish or Reject. Of course, generosity is gauged relative to available opportunities. Thus the property right $m_{0}$ will differ across the four games, and the rejection rate of a particular offer will be higher when it is less generous relative to the alternative offer. 
Our empirical analysis of the FFF data begins with coding responder choice by

$$
Z= \begin{cases}0, & \text { if } S \text { chooses }(0,0) \\ 1, & \text { otherwise }\end{cases}
$$

We construct the explanatory variables according to assumptions A.1 and A.2 as follows. The status variable $s$ is constant here because the FFF data contain no variation in status (other than relative payoff), so $s=s_{F}-s_{S}=0$. Take a first-order Taylor expansion of the expression in A.2 to write an individual's emotional state as $\theta_{i}=\theta_{0}+a r(x)+\epsilon_{i}$, where the coefficient $a$ is the responsiveness to reciprocity. As in the previous application, we assume that the density for $\epsilon_{i}$ is given in equation (2).

A key empirical issue is how the property right $m_{0, k}$ varies across the four games $(10-k) / k$ for $k=0,2,5$, and 8 . Some authors specify property rights a priori, but data provide direct evidence, so we instead estimate property rights. For $x=$ "Take" the reciprocity variable is $r(x)=2-m_{0, k}$ and for $x=$ "Share" it is $r(x)=m(x)-m_{0, k}$ where $m(x)=k$ in game $k$. As in the Stackelberg application, residual benevolence $\theta_{0}$ cannot be identified separately from the property rights $m_{0, k}$, so we absorb $\theta_{0}$ into each $m_{0, k}$ term. Tests of the hypotheses that $a=0$ and that $m_{0, k}=m_{0, j}$ are unaffected by this absorption.

Assumption A.1 implies that the first mover action $x$ will be accepted $(Z=1)$ if and only if $0=u(0,0)<u(m(x), y(x))=\frac{1}{\alpha}\left(m(x)^{\alpha}+(\theta+\epsilon) y(x)^{\alpha}\right)$, and the presence of rejections in the data precludes a negative value of $\alpha \cdot{ }^{10}$ So the acceptance condition is $0<(m / y)^{\alpha}+a r(x)+\epsilon$ or $-\epsilon<(m / y)^{\alpha}+a r(x)$. Hence the probability that $Z=1$ is given by the cumulative error function distribution $F(z ; b, c)$ evaluated at $z=(m / y)^{\alpha}+\operatorname{ar}(x)$.

The likelihood function is formed by writing down the probability of the data set as a function of the utility model parameters $\alpha, a, m_{0,0}, m_{0,2}, m_{0,5}$, and $m_{0,8}$, and the error function parameters $b$ and $c$. For the $5 / 5$ game, for example, the log likelihood of the observed data (25 Tolerate, 20 Punish, 45 Accept, and 0 Reject) is

$$
\begin{aligned}
\ln L_{5}\left(\alpha, a, m_{0,5}, b, c\right)= & 25 \ln F\left((1 / 4)^{\alpha}+a\left(2-m_{0,5}\right) ; b, c\right) \\
& +20 \ln \left(1-F\left((1 / 4)^{\alpha}+a\left(2-m_{0,5}\right) ; b, c\right)\right) \\
& +45 \ln F\left(1+a\left(5-m_{0,5}\right) ; b, c\right) .
\end{aligned}
$$

\footnotetext{
${ }^{10}$ As in the dictator game in Section 4 , utility is $-\infty$ if $\alpha<0$ and either $m=0$ or $y=0$.
} 
Define the $\log$ likelihood similarly for $k=0,2$, and 8 . Then the log likelihood function is

$$
\ln L\left(\alpha, a, m_{0,0}, m_{0,2}, m_{0,5}, m_{0,8}, b, c\right)=\sum_{k \in\{0,2,5,8\}} \ln L_{k}\left(\alpha, a, m_{0, k}, b, c\right)
$$

for the experiment data sample. ${ }^{11}$ Table 3 shows parameter estimates and standard errors.

\begin{tabular}{ccc} 
Parameter & Estimate & SE \\
\hline$\alpha$ & 0.095 & 0.549 \\
$a$ & 1.268 & 0.755 \\
$m_{0,0}$ & 1.308 & 0.273 \\
$m_{0,2}$ & 1.607 & 0.329 \\
$m_{0,5}$ & 2.504 & 0.504 \\
$m_{0,8}$ & 1.906 & 0.366 \\
$b$ & 2.133 & 1.142 \\
$c$ & 0 & \\
\hline
\end{tabular}

Table 3: Parameter estimates and standard errors from mini-ultimatum games

We test hypothesis H.1 by examining the null hypothesis that the reciprocity sensitivity parameter $a$ is zero. For this hypothesis test, we write $\theta=\theta_{0}+a\left(x-\tilde{m}_{0, i}\right)$ for $i=0,2,5$, and 8 and find the $\log$ likelihood for the restricted model with $\theta=\theta_{0}$. The likelihood ratio test statistic for the restricted model is $\chi^{2}(1)=18.02$, firmly rejecting the null hypothesis at a $p$-value of less than $10^{-4}$, in favor of H.1. Thus application of our model to the FFF mini-ultimatum game data leads to the conclusion that subjects exhibit reciprocal behavior.

We also test the hypotheses that $m_{0,0}=m_{0,5}$ and $m_{0,2}=m_{0,5}$. Since equal payoffs are possible in the $5 / 5$ game, but not in either the $10 / 0$ game or the $8 / 2$ game, rejection of these hypotheses provides support for context dependent property rights. The $\chi^{2}$ statistic for the hypothesis that $m_{0,0}=m_{0,5}$ is $\chi^{2}(1)=15.56$, which has $p$-value less than 0.0001 . The $\chi^{2}$ statistic for the hypothesis that $m_{0,2}=m_{0,5}$ is $\chi^{2}(1)=10.51$, which has $p$-value less than 0.0012. These results provide evidence, contrary to pure distributional models, that second movers' perceived property rights in mini-ultimatum games change as the set of first mover alternatives changes.

We conclude from this application that reciprocity has a major impact on second mover behavior in mini-ultimatum games as well as in Stackelberg games. In addition, the set of

11 One second mover rejected an offer of 8 in the $2 / 8$ game. This choice has not been included in the likelihood function $L_{8}\left(\alpha, a, m_{0,8}, b, c\right)$ because it distorts the estimates and it probably resulted from confusion. Parameter estimates are obtained from the remaining 359 second mover choices. 
available alternatives clearly affects perceived property rights, and these perceived rights affect behavior.

\section{$7 \quad$ Status and reciprocity in ultimatum games}

Hoffman, McCabe, Shachat, and Smith (1994, HMSS hereafter) report ultimatum games with several treatments that include status variables. The baseline treatment $R$ has random role assignment, in which it is natural to assign status variables zero to both the first and second movers. The contest role assignment treatment $S$ assigns individual subjects to first and second mover roles based on their performance on a quiz: subjects with scores in the top half are assigned to the first mover role. Since the advantageous first mover role is "earned" in the contest assignment treatment, the second mover's perceived entitlement may shrink. To test this conjecture, it is natural to set first mover status to 1 in this treatment and keep second mover status at 0 .

In the HMSS ultimatum game, the first mover proposes an allocation $x \in\{0,1,2, \ldots, 10\}$ for the second mover and $10-x$ for himself. The second mover then either accepts that proposal, yielding the outcome $(m, y)=(x, 10-x)$, or else rejects it, yielding $(m, y)=(0,0)$. The HMSS data include only proposals $x \in\{2,3,4,5\}$ : the other possible proposals were never observed in either treatment. The data consist of four ordered pairs for each treatment: the number of proposals and the number of rejections for each of the four observed values of $x$. In the random treatment, the data are $R=((0,0),(3,1),(9,1),(12,0))$. In the status (or quiz) treatment, the data are $S=((1,0),(9,0),(12,0),(2,0))$.

Our analysis of this game combines a test of reciprocity with the part of H.1 not testable in the earlier data sets: whether the status variable $s$ has a positive impact on acceptance rates. To begin, set $s=s_{F}-s_{S}=1-0=1$ in treatment $S$ and $s=s_{F}-s_{S}=0-0=0$

in treatment $R$. Denote the neutral first mover choices in the two treatments by $x_{0}^{R}$ and $x_{0}^{S}$, and note that the second mover's maximum payoff given the first mover choice $x$ is $m(x)=x$ (obtained by accepting the proposal). The reciprocity variable can be expressed as $r(x)=m(x)-m\left(x_{0}^{t}\right)=x-x_{0}^{t}$ in treatment $t=R, S$, which has the general expression $r(x)=x-x_{0}^{R}(1-s)-x_{0}^{S} s$. From the usual first-order Taylor series expansion, and 
absorption of $\theta_{0}$ into the neutral choices (or property rights), the emotional state function becomes $\theta(r, s)=a\left(x-x_{0}^{R}(1-s)-x_{0}^{S} s\right)$.

As in the MUG data analysis, code acceptance of proposal $x$ by the second mover as $Z(x)=1$ and code rejection as $Z(x)=0$. Once again, assumption A.1 implies that $Z(x)=1$ if and only if $u_{i}(x, 10-x)>u_{i}(0,0)$ for second mover $i$, and the presence of some rejections in the data preclude a negative value of $\alpha$. Thus we have $u_{i}(0,0)=0$ and $u_{i}(x, 10-x)=\frac{1}{\alpha}\left(x^{\alpha}+\left(a\left(x-x_{0}^{R}(1-s)-x_{0}^{S} s\right)+\epsilon_{i}\right)(10-x)^{\alpha}\right)$. It follows directly that $Z(x)=1$ whenever $x^{\alpha}+\left(a\left(x-x_{0}^{R}(1-s)-x_{0}^{S} s\right)+\epsilon_{i}\right)(10-x)^{\alpha}>0$ or

$$
-\epsilon_{i}<(x /(10-x))^{\alpha}+a\left(x-x_{0}^{R}(1-s)-x_{0}^{S} s\right) .
$$

As in the previous applications, we assume that the individual subject idiosyncrasy variable $\epsilon_{i}$ has the error function distribution with the density in equation (2). Then the probability that $Z(x)=1$ may be written in terms of the model parameters as

$$
P\left(x ; \alpha, a, x_{0}^{R}, x_{0}^{S}, s, b, c\right) \equiv F\left(a\left(x-x_{0}^{R}(1-s)-x_{0}^{S} s\right)+(x /(10-x))^{\alpha} ; b, c\right),
$$

where once again $F(z ; b, c)$ is the CDF for the error function.

To construct the log likelihood function, consider the data for $x=3$. In the $R$ (or $s=0$ ) treatment there are two acceptances and there is one rejection, and in the $S$ (or $s=1$ ) treatment there are nine acceptances and there is no rejection. Hence

$$
\begin{aligned}
\ln L_{3}\left(\alpha, a, x_{0}^{R}, x_{0}^{S}, b, c\right)= & 2 \ln P\left(3 ; \alpha, a, x_{0}^{R}, x_{0}^{S}, 0, b, c\right) \\
& +\ln \left(1-P\left(3 ; \alpha, a, x_{0}^{R}, x_{0}^{S}, 0, b, c\right)\right) \\
& +9 \ln P\left(3 ; \alpha, a, x_{0}^{R}, x_{0}^{S}, 1, b, c\right) .
\end{aligned}
$$

Using analogous definitions for the other observed offers $x=2,4$, and 5 we obtain

$$
\ln L\left(\alpha, a, x_{0}^{R}, x_{0}^{S}, b, c\right)=\sum_{j=2}^{5} \ln L_{j}\left(\alpha, a, x_{0}^{R}, x_{0}^{S}, b, c\right) .
$$

Parameter estimates and standard errors are shown in table 4. Although the standard error for $a$ is extremely small, that parameter is highly correlated with $\alpha, x_{0}^{S}$, and $b$, so uncertainty about that parameter value enters through the correlation.

Now we are prepared to test both parts of hypothesis H.1. First, as with the Stackelberg and MUG data, we test the null hypothesis that second movers don't respond appropriately to reciprocity and status concerns, i.e., the null hypothesis that $a=0$. Second, we want to 


\begin{tabular}{ccc} 
Parameter & Estimate & SE \\
\hline$\alpha$ & 0.346 & 3.485 \\
$a$ & 0.520 & 0.000 \\
$x_{0}^{R}$ & 3.507 & 0.967 \\
$x_{0}^{S}$ & 0.408 & 1.028 \\
$b$ & 1.447 & 2.457 \\
$c$ & 0 & \\
\hline
\end{tabular}

Table 4: Parameter estimates and standard errors from ultimatum games

ascertain whether the neutral (or reference) offer expected by second movers decreases when the status of the first mover increases. That is, we test the null hypothesis that $x_{0}^{R}=x_{0}^{S}$.

The $\log$ likelihood at the parameter estimates is $\ln L=-5.049$. With the restriction $a=0$, the $\log$ likelihood is $\ln L=-7.574$. The likelihood ratio test statistic is $2(7.574-5.049)=5.05$, which has $p$-value 0.025. For the restricted model with $x_{0}^{R}=x_{0}^{S}$, the test statistic is 5.114 which yields $p$-value 0.024 . Thus we can reject both null hypotheses at better than the $5 \%$ level in favor of H.1, that the emotional state function increases in status $s$ as well as in reciprocity $r$.

This application reveals the significance of status as a determinant of behavior in ultimatum games. It also demonstrates that our structural estimation strategy robustly accommodates individual-subject idiosyncrasies.

\section{Further applications}

This section describes three additional applications of the model, using the same empirical techniques. The first application returns to the dictator game in Cherry, Frykblom, and Shogren (2002) and interprets their earned endowment treatment as a status variable. The next application considers dictator control data from the investment game in Cox (2004), and looks for efficiency concerns. The final application is to the relatively complex moonlighting game data from Cox, Sadiraj, and Sadiraj (2004) which reinforces previous results on reciprocity.

\section{Status in a dictator game}

The dictator game in Cherry, Frykblom, and Shogren (2002) provides additional evidence that supports the importance of status. In their earnings treatment, roles are assigned ran- 
domly, but dictators take a quiz with 17 sample questions from the Graduate Management Admissions Test (GMAT). Those who answer at least ten questions correctly receive an endowment of $\omega=\$ 40$; the others recieve an endowment of $\omega=\$ 10$. Choice frequencies in the $\omega=\$ 10$ game with earned endowment are $h_{10}=(22,2,2,2)$ for $x \in\{0,1,2,3\}$ (compared to $g_{10}=(6,3,4,4,3,6)$ for $x \in(0,1,2, \ldots, 5)$ in the baseline treatment). A comparable shift in offers occurred in the $\omega=\$ 40$ game.

With status represented as an indicator variable when dictators' earn the endowment, the dictator's weight $\theta$ on the other player's payoff can be modeled as $\theta_{i}=\theta_{0}-a s+\epsilon_{i}$. The maximization problem can be framed in terms of the utility function parameters $\alpha, \theta_{0}$, and $a$ and the error term distribution parameters $b$ and $c$ as

$$
\max _{x} u\left(x ; \alpha, a, \theta_{0}, b, c\right)=\max _{x}\left((\omega-x)^{\alpha}+\left(\theta_{0}-a s+\epsilon\right) x^{\alpha}\right) .
$$

\begin{tabular}{ccc} 
Parameter & Estimate & SE \\
\hline$\alpha$ & 0.075 & 0.174 \\
$\theta_{0}$ & 0.337 & 0.082 \\
$a$ & 0.460 & 0.097 \\
$b$ & 0.735 & 0.081 \\
$c$ & 0 & \\
\hline
\end{tabular}

Table 5: Parameter estimates and standard errors from dictator game with status treatment

Parameter estimates and standard errors, with offer probabilities obtained as in Section 4 , are shown in table 5 . The constrained model, with $a=0$, has likelihood -211.123 , whereas the unconstrained model has likelihood -193.648 , so the test statistic for the hypothesis that $a=0$ is $\chi^{2}(1)=34.95$. Thus at a microscopic $p$-value, we reject the hypothesis that $a=0$ in favor of the research hypothesis $a>0$, i.e., the dictator's weight $\theta$ on the other's monetary payoff decreased when she earned a higher endowment. Comparison to the application in Section 4 reveals the importance of status considerations for explaining behavior even in simple distribution games.

\section{Preference for efficiency in a dictator game}

The dictator game from Cox (2004) provides direct evidence that our model captures a concern for efficiency. Players in this game start with the endowment $(m, y)=(10,10)$. Following the transfer by the dictator, the passive recipient receives three additional units 
of payoff for each unit transferred by the dictator; thus positive transfers increase efficiency. If the number of dictators who transferred $i$ units (for $i$ between zero and ten) is denoted $g_{i}$, then the data are represented by the vector $g=(11,3,1,2,1,3,1,1,1,2,4)$. The estimation procedure for this game is similar to the one used in Section 4 for the baseline dictator game from Cherry, Frykblom, and Shogren. Parameter estimates and standard errors for this game are shown in table 6 .

\begin{tabular}{ccc} 
Parameter & Estimate & $\mathrm{SE}$ \\
\hline$\alpha$ & 0.792 & 0.067 \\
$\theta_{0}$ & 0.349 & 0.085 \\
$b$ & 0.043 & 0.001 \\
$c$ & 3.411 & 0.718 \\
\hline
\end{tabular}

Table 6: Parameter estimates and standard errors from dictator game

The probability distribution over choices that results from these parameter estimates implies an average transfer from the second mover (or dictator) of 4.08 units to the first mover, which represents a substantial preference for efficiency compared to self-regarding (or "economic man") preferences.

\section{Reciprocity in the moonlighting game}

The moonlighting game introduced by Abbink, Irlenbusch, and Renner (2000) demonstrates the flexibility of our model and our structural estimation procedure. Cox, Sadiraj, and Sadiraj (2004) conducted sessions with 30 pairs of first and second movers; the data are shown in figure 7 . The game begins with the endowment $(m, y)=(10,10)$ for players $S$ and $F$. The first mover then selects an amount $x \in\{-5,-4,-3, \ldots, 10\}$ to take from or give to the second mover. Positive transfers by the first mover are tripled. The interim allocations that result from these first mover choices are shown as circles in figure 7 . All lie on the first mover budget set formed from the two line segments between the points $(5,15)$, $(10,10)$, and $(40,0)$. Second movers then select an amount $y$ to transfer to or take from the first mover. This can be any positive amount less than or equal to the amount that the second mover has available, or it can be negative. A negative transfer by the second mover represents a penalty that reduces first mover payoff by three times the amount transferred (but does not make it negative). Final allocations that follow second mover choices are 
shown as squares in the figure (unless there is no transfer by the second mover, in which case it remains at the interim allocation).

This game has complicated choice sets, but our general procedure still applies. The normalized reciprocity function is $r(x)=\left(10+(1+2 \operatorname{Sign}(x))|x|-m_{0}\right) / 35$ and the second mover's weight on first mover payoff is $\theta_{i}=\operatorname{ar}(x)+\epsilon_{i}$. As usual, the key step in the estimation procedure is to determine - for given utility function parameters and error term parameters - the probability of each choice that is available to $S$. To illustrate, suppose that the first mover chooses $x=1$. The interim allocation then is $(m, y)=(13,9)$, and $S$ chooses an allocation from the set $\{(13-y, 9+y)\}_{y=0}^{13} \cup\{(13-y, 9-3 y)\}_{y=0}^{3}$. This choice set is more complicated than in the dictator or Stackelberg games, but the procedure again is to determine threshold values of $\epsilon$ that correspond to each possible choice. The choice probabilities applied to the data determine the likelihood function.

Much of the data lies at corners and kinks of the second mover's opportunity set. This is predicted by our model: for a range of values of $\theta$ in a neighborhood of zero the optimal second mover transfer is zero. This occurs frequently for negative or low values of the first mover transfer $x$, and much less frequently for larger values of $x$, since these induce a higher value for the reciprocity function $r(x)$.

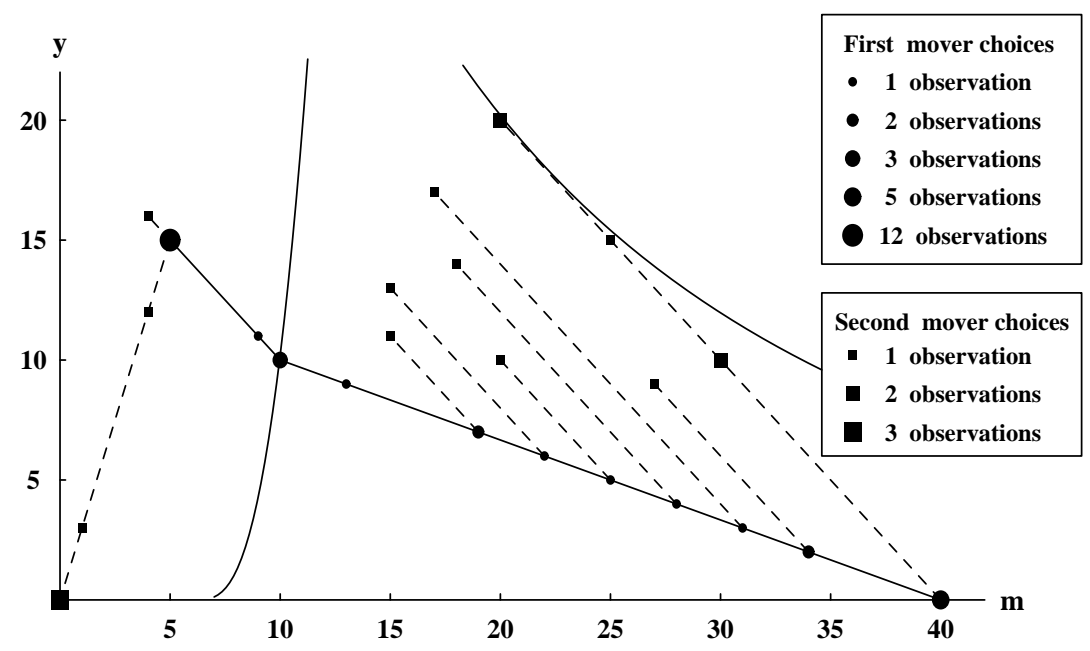

Figure 7: Interim and final allocations in the moonlighting game.

There are two data points which our model does not predict. When the first mover chooses $x=-5$, then one portion of the second mover budget set is the line segment 
between $(0,0)$ and $(5,15)$. Figure 1 (b) shows that indifference curves for $\theta<0$ can't be tangent to this line segment, hence the model predicts that the data will cluster at the points $(0,0)$ and $(5,15)$. The data points at $(1,3)$ and $(4,12)$ differ by one unit from these corners; to expedite the analysis we move them to the corners. We also moved a third stray data point - a gift of one unit by a second mover after the first mover took five units - to $(5,15)$ on the presumption that it was an error (or possibly an ironic gesture) by the second mover, not an expression of genuine altruism. Parameter estimates and standard errors are shown in table 7 . The $p$-value for the hypothesis test that the reciprocity parameter $a=0$ is $p=0.0694$. Given the relatively small data set, we are able to reject the hypothesis at a reasonable level.

\begin{tabular}{ccc} 
Parameter & Estimate & $\mathrm{SE}$ \\
\hline$\alpha$ & 0.286 & 0.166 \\
$a$ & 1.184 & 0.140 \\
$m_{0}$ & 13.97 & 0.331 \\
$b$ & 0.678 & 0.140 \\
$c$ & 0 & \\
\hline
\end{tabular}

Table 7: Parameter estimates and standard errors from moonlighting game

Estimated indifference curves passing through $(10,10)$ and $(20,20)$ in figure 7 correspond to offers $x=0$ and $x=10$. The curves show that the emotional state of the typical second mover switches from slightly negative to very positive over this range of first mover choices, indicating once again that reciprocity plays a major role. Thus the model captures the tendency of second movers to reward first movers who make higher offers.

\section{Discussion}

We hypothesize that a person's desire to help or harm others depends on emotional states that arise from a reciprocity motive and from status considerations. In this paper we propose a simple parametric model incorporating this hypothesis.

The first hurdle for an empirical model is tractability: can the model be estimated from available data? We obtained an affirmative answer by applying a structural estimation procedure to six existing data sets. 
The baseline treatment from the first data set, a dictator game, illustrates our basic model of other-regarding preferences. Dictators' choices exhibit residual altruism, independently of reciprocity motives or status considerations. The estimates indicate uniformly distributed individual idiosyncracies for greater or lesser altruism. The earned endowment treatment in the same data set provides evidence that status significantly affects the emotional state function $\theta$, which determines the weight placed on the other player's payoff.

The second data set, a Stackelberg duopoly game, involves output choices from a range of values by a first and second mover. Parameter estimates indicate strong reciprocity, which is primarily negative but positive as well for low first-mover output (as in figure 5 (a)). Here and elsewhere, reciprocity better accounts for the data than simple inequality aversion. The third data set, mini-ultimatum games, consists of binary choices by second movers following binary choices by first movers. The parameter estimates and tests of hypotheses show that unchosen alternatives affect perceived property rights, and that choices reflect reciprocity based on these perceived rights.

The fourth data set, an ultimatum game with a status treatment, demonstrates that status systematically alters the property right or reference payoff of the second mover. The fifth data set, from a dictator control treatment for the investment game, demonstrates that decision makers have a preference for efficiency. Finally, the moonlighting game demonstrates the flexibility of our estimation procedure and provides additional evidence that distributional preferences are conditional on reciprocity.

Of course, to be considered successful and important, an empirical model must jump further hurdles. Does the model suggest new experimental designs? Can extensions deal with different sorts of data? How does the model compare to alternative models? We close with a few thoughts on these matters.

The model does suggest new experimental designs. For example, consider two player extensive form game experiments that elicit willingness to pay ( WTP) own payoff for other's payoff, while systematically varying relative income opportunities $y / m$, other aspects of status $s$, and reciprocity considerations $r$. The data would allow sharp estimates for the impact of each motive.

In our applications that involve reciprocity, we estimated property rights $m_{0}$ (with resid- 
ual benevolence $\theta_{0}$ absorbed where necessary). This suggests the need for new experimental designs that develop an empirically grounded, general specifications of property rights $m_{0}$ and that allow separate identification of residual benevolence $\theta_{0}$. In particular, we propose asking subjects (behind the veil of ignorance) what they think the second mover can reasonably expect. In games with a strong consensus on the property right we expect to see a relatively large coefficient on $r$. When there is little consensus one might expect a larger self-serving bias.

Assumption A.2 states that individuals differ only in idiosyncratic additive components of the emotional state variable $\theta$. Some evidence suggests that people may differ in their responsiveness $a$ to reciprocity and status considerations. Therefore future work should consider estimation using random coefficient models.

Future work should explore games with more than two players. The model extends directly. My utility function depends on every other player $i$ 's payoff $y_{j}$, via my emotional attitude $\theta_{j}$ towards player $j$, and my utility function is simply

$$
u\left(x, y_{1}, \ldots, y_{n}\right)=\alpha^{-1}\left(x^{\alpha}+\theta_{1} y_{1}^{\alpha}+\ldots+\theta_{n} y_{n}^{\alpha}\right) .
$$

The natural extension of assumption A.2 is that $\theta_{j}=\theta\left(r_{j}, s_{j}\right)+\epsilon_{j}$, i.e., the emotional attitude depends on player-pair specific reciprocity experience $r_{j}$ and relative status $s_{j}$. Of course, in games where players can't separately identify the other players, there is only one $\theta$. For games in which each player can observe the individual history of every other player, the model could be enriched to include an indirect reciprocity motive as well as the direct motive captured in $r$.

Once other-regarding preference models are thoroughly explored, it may be worthwhile to use them to construct complete models of behavior in extensive form games of perfect information. It is natural to assume that the first mover maximizes the expectation of a utility function that has the same form as the second mover's utility function, as in the dictator game application in Section 4.

Such an extension, however, raises two new issues. First, what are the first mover's beliefs regarding second mover behavior? ${ }^{12}$ One could, for example, assume that the first mover acts as if she knows the empirical distribution of second mover behavior, or at least

12 The empirical importance of such beliefs is supported by significant differences between first mover 
its mean. Second, and more distinctively for our model, does the first mover's $\theta$ function respond to prospective reciprocity? One could set $r=0$ since the second mover hasn't actually done anything at the time the first mover makes her choice. Departures from selfish optimality (i.e., $\theta \neq 0)$ then would be attributed to status considerations $(s \neq 0)$ and/or residual benevolence or malevolence $\left(\theta_{0} \neq 0\right)$ in addition to expectations about prospective second mover changes in $m$ and $y$. But it is likely in multi-round experiments with fixed matching and possible in random re-matching experiments that a first mover's experiences in earlier matchings will affect his emotional state in a manner best captured by $r \neq 0$.

The equilibrium models based on psychological games impose strong assumptions regarding both issues. First, they assume that expectations are based on equilibrium beliefs (direct and higher order). Second, they implicitly assume that prospective second mover behavior produces as strong an emotional response as actual behavior. We hesitate to impose such strong assumptions a priori. Both issues, we believe, should be resolved empirically.

The same two issues arise in normal form games, in games of incomplete information, and in games where a given player has several moves. When these two issues are resolved, we believe our approach can be extended to such games.

Our approach has several advantages that might survive beyond the current implementation. First of all, it uses a model of preferences and choice, not equilibrium, and so is tractable and extensible. Second, it is more flexible than unconditional distributional preference models in that it takes other motives into account. Third, it is open to new findings in the psychology of emotions and so may facilitate interdisciplinary cross-fertilization.

behavior in investment and trust games and in dictator control treatments with the same respective feasible sets and message spaces (Cox, 2004; Cox and Deck, 2005). 


\section{Appendix A.1: Utility function for $\alpha=0$}

Since we use a CES utility function with the factor $1 / \alpha$ rather than the exponent $1 / \alpha$, we verify that the limit utility function is also a Cobb-Douglas utility function for our alternative specification. Let

$$
u(m, y ; \alpha)= \begin{cases}\frac{1}{\alpha}\left(m^{\alpha}+\theta y^{\alpha}\right), & \alpha \in(-\infty, 0) \cup(0,1], \\ m y^{\theta}, & \alpha=0 .\end{cases}
$$

We want to show that for $\alpha \neq 0$, the indifference curves of $u(m, y ; \alpha)$ converge to indifference curves of $u(m, y ; 0)=m y^{\theta}$. Fix a point $\left(m_{0}, y_{0}\right)$ with $m_{0}>0$ and $y_{0}>0$. For every $\alpha \in(-\infty, 0) \cup(0,1]$, the set $\left\{(m, y): u(m, y ; \alpha)=u\left(m_{0}, y_{0} ; \alpha\right)\right\}$ is the indifference curve for the given $\alpha$ that passes through the point $\left(m_{0}, y_{0}\right)$. On this indifference curve, $y$ can be written as a function of $m$ :

$$
y(m ; \alpha)=\left(\frac{m_{0}^{\alpha}+\theta y_{0}^{\alpha}-m^{\alpha}}{\theta}\right)^{1 / \alpha} .
$$

It suffices to show for each fixed $\bar{m}>0$ that $y(\bar{m} ; \alpha)$ converges pointwise to $y(\bar{m} ; 0)=m_{0}^{1 / \theta} y_{0} \bar{m}^{-1 / \theta}$ as $\alpha \rightarrow 0$.

The limit of $y(\bar{m} ; \alpha)$ as $\alpha \rightarrow 0$ can be determined by applying L'Hospital's rule to $\ln y(\bar{m} ; \alpha)$ :

$$
\begin{aligned}
\lim _{\alpha \rightarrow 0} \ln y(\bar{m} ; \alpha) & =\lim _{\alpha \rightarrow 0} \frac{1}{\alpha} \ln \left(\frac{m_{0}^{\alpha}+\theta y_{0}^{\alpha}-\bar{m}^{\alpha}}{\theta}\right) \\
& =\lim _{\alpha \rightarrow 0} \frac{m_{0}^{\alpha} \ln m_{0}+\theta y_{0}^{\alpha} \ln y_{0}-\bar{m}^{\alpha} \ln \bar{m}}{\theta} .
\end{aligned}
$$

From this it follows that

$$
\lim _{\alpha \rightarrow 0} \ln y(\bar{m} ; \alpha)=\frac{\ln m_{0}+\theta \ln y_{0}-\ln \bar{m}}{\theta}
$$

so that it is natural to define

$$
\ln y(\bar{m} ; 0) \equiv \frac{\ln m_{0}+\theta \ln y_{0}-\ln \bar{m}}{\theta} .
$$

Along the indifference curves of $u(m, y ; 0), m y^{\theta}=m_{0} y_{0}^{\theta}$, which is the required result.

\section{Appendix A.2: Construction of dictator game likelihood function}

The key idea in the formulation of the likelihood function for the choice of the amount $x$ sent by the dictator is that for parameter values $\alpha$ and $\theta_{0}$, the utility maximizing choice of $x$ is a function of the idiosyncratic error $\epsilon$. As $\epsilon$ varies there are critical values where the utility maximizing choice changes, and the range of values of $\epsilon$ that support a given choice determine the probability of that choice through the distribution function for $\epsilon$.

Suppose that $\epsilon$ has the error function distribution in equation (2). For fixed values of the dictator utility function parameters, the optimal choice of $x$ depends only on $\epsilon$. The values of $x$ are in the 
set $\{0,1,2, \ldots, 10\}$, so we want to determine threshold values $\epsilon_{x}$ such that $u\left(x, ; \alpha, \theta_{0}, \epsilon_{x}, b, c\right)=$ $u\left(x+1 ; \alpha, \theta_{0}, \epsilon_{x}, b, c\right)$. For $\epsilon \in\left(-\infty, \epsilon_{0}\right)$, the optimal choice is $x=0$. For each $x \in\{1,2,3, \ldots, 9\}$, when $\epsilon \in\left(\epsilon_{x-1}, \epsilon_{x}\right)$, the optimal choice is $x$. When $\epsilon \in\left(\epsilon_{9}, \infty\right)$ the optimal choice is $x=10$. The probability of $x$ can therefore be determined from the cumulative distribution function $F(z ; b, c)$ of the error function distribution.

The probability of $x=0$ is $F\left(\epsilon_{0} ; b, c\right)$. For $x \in\{1,2,3, \ldots, 9\}$ the probability of $x$ is $F\left(\epsilon_{x}, b, c\right)-$ $F\left(\epsilon_{x-1} ; b, c\right)$. Finally, for $x=10$, the probability of $x$ is $1-F\left(\epsilon_{9} ; b, c\right)$. These probabilities determine the likelihood function

$$
L\left(x ; \alpha, \theta_{0}, b, c\right)=\prod_{i=1}^{52} \operatorname{Pr}\left[x_{i}=x ; \alpha, \theta_{0}, \epsilon_{x}, b, c\right]
$$

and maximum likelihood estimates are obtained by maximizing this function (or its logarithm).

Standard error estimates are obtained from the numerical Hessian matrix, as the square roots of its diagonal elements. For details on this estimator, see pages 260 to 266 of Davidson and MacKinnon (1993).

\section{Appendix A.3: Definition of the Stackelberg best-response function $q^{*}(x ; \theta, \alpha)$}

The second mover best-response function in the Stackelberg game cannot be solved in closed form, except in a few special cases. The purpose of this appendix is to demonstrate that nevertheless the best-response function is well-defined and has a unique maximizer for all values of the parameters.

Theorem 1: For each $x \in(0,24)$ and each $(\theta, \alpha) \in(-\infty, \infty) \times(-\infty, 1]$ there is a unique $q^{*} \in(0,24-x]$ that maximizes the utility function

$$
U(x, q)= \begin{cases}\frac{1}{\alpha}(24-x-q)^{\alpha}\left(q^{\alpha}+\theta(x, \epsilon) x^{\alpha}\right), & \alpha \neq 0, \\ (24-x-q)^{1+\theta(x, \epsilon)} q x^{\theta(x, \epsilon)}, & \alpha=0 .\end{cases}
$$

Proof: We partition the space of values for $\theta, \alpha$, and $x$ into a connected (relatively) open set $A$ with a unique interior solution to the utility maximization problem and into a connected closed set $B$ with a boundary solution to the utility maximization problem. The boundary between sets $A$ and $B$, which depends on $\theta, \alpha$, and $x$, is characterized by the function $\theta(x, \alpha)=-\left(\frac{24-x}{x}\right)^{\alpha}$. Region $B$ is subdivided into regions $B_{1}$ and $B_{2}$. In region $B_{1}$ the utility function is bounded because $\frac{1}{\alpha}(24-x-q)^{\alpha}\left(q^{\alpha}+\theta(x, \epsilon) x^{\alpha}\right)$ is bounded for $\alpha>0$. In region $B_{2}$ the utility function has an asymptote as $q \rightarrow 24-x$. Figure A.3.1 (a) shows these three regions in a cross section for $x=8$; figure A.3.1 (b) shows a cross section for $x=16$.

In order to evaluate properties of $U(x, q)$, it is useful to represent the output $q$ of $S$ as $q=c(24-x)$ with $c \in[0,1]$. Define $\tilde{U}_{x}(c) \equiv U(x, c(24-x))$. Then

$$
\tilde{U}_{x}(c)=\frac{1}{\alpha}(1-c)^{\alpha}(24-x)^{\alpha}\left(c^{\alpha}(24-x)^{\alpha}+\theta x^{\alpha}\right) .
$$




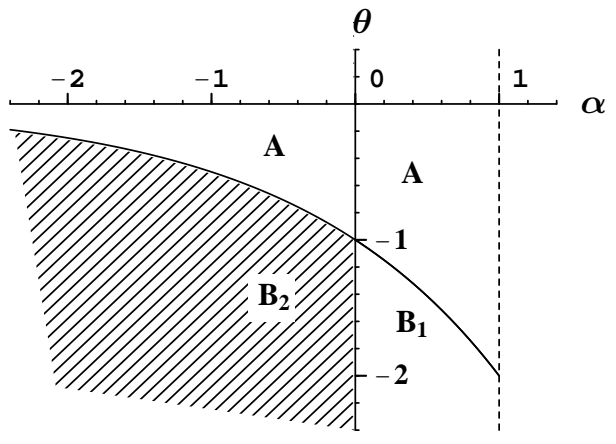

(a) $\theta(x, \epsilon)$ for $x=8$

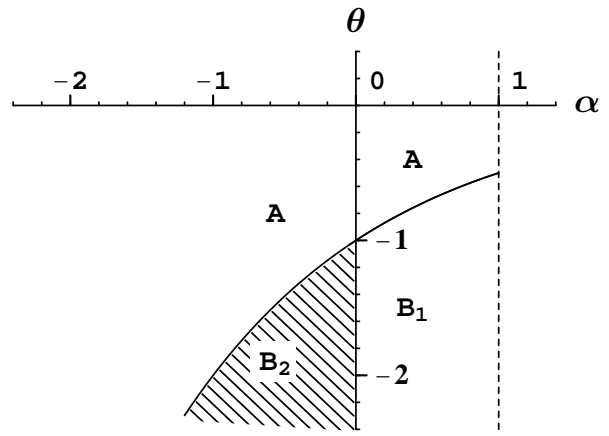

(b) $\theta(x, \epsilon)$ for $x=16$

Figure A.3.1: Regions evaluated to characterize the best response function.

The derivative of this utility function is

$$
\tilde{U}_{x}^{\prime}(c)=(1-c)^{\alpha-1}(24-x)^{\alpha}\left((1-2 c) c^{\alpha-1}(24-x)^{\alpha}-\theta x^{\alpha}\right) .
$$

For $c \in(0,1), \tilde{U}_{x}^{\prime}(c)=0$ if and only if $f_{x}(c) \equiv(1-2 c) c^{\alpha-1}(24-x)^{\alpha}-\theta x^{\alpha}$ is zero. Lemma 1 shows that for $\alpha \in[-2,0) \cup(0,1), f_{x}(c)$ is a convex function, so that $f_{x}(c)$ has at most two roots. Lemma 2 shows that for $\alpha \in(-\infty,-2), f_{x}(c)$ is convex on an interval $\left(0, c^{\prime}\right)$ and monotonically increasing on $\left(c^{\prime}, 1\right)$, so that it again has at most two roots. The two lemmas are used subsequently to prove Claims 1 through 3, which show that there is a unique maximizer of $\tilde{U}_{x}(c)$ for regions $A$, $B_{1}$, and $B_{2}$. Claims 4 and 5 treat the cases $\alpha=0$ and $\alpha=1$ separately, but shows that they are consistent with the other cases. Theorem 1 follows from Claim 1 through 5.

Lemma 1: For $\alpha \in[-2,0) \cup(0,1), f_{x}(c)$ is a convex function of $c$, for all $c \in(0,1)$. Therefore the first-order condition for a local maximum, $\tilde{U}_{x}^{\prime}(c)=0$, has at most two roots in $(0,1)$ for these values of $\alpha$.

Proof: For $\alpha \in(-\infty, 0) \cup(0,1], f_{x}^{\prime \prime}(c)=(\alpha-1) c^{\alpha-3}(24-x)^{\alpha}(\alpha-2 \alpha c-2)$. For $\alpha<1$, this has the opposite sign from the last term, so $f_{x}(c)$ is convex when $\alpha-2 \alpha c-2<0$. For $\alpha \in(0,1)$ and for $\alpha \in[-2,0)$ this inequality holds for all $c \in(0,1)$. Hence for $\alpha \in[-2,0) \cup(0,1), f_{x}(c)$ is convex.

Lemma 2: For $\alpha \in(-\infty,-2), f_{x}(c)$ is a convex function of $c$ for $c \in\left(0, \frac{\alpha-2}{2 \alpha}\right)$, and it is a strictly increasing function for $c \in\left(\frac{\alpha-2}{2 \alpha}, 1\right)$. Therefore, $f_{x}(c)$ has at most two roots on $(0,1)$.

Proof: As noted in the proof of Lemma 1, $f_{x}(c)$ is convex only if $\alpha-2 \alpha c-2<0$. For $\alpha<0$ this is equivalent to the inequality $c<\frac{\alpha-2}{2 \alpha}$, and if $\alpha<-2$, then $\frac{\alpha-2}{2 \alpha}<1$ so that convexity only holds for $c \in\left(0, \frac{\alpha-2}{2 \alpha}\right)$. Since $f_{x}^{\prime}(c)=c^{\alpha-2}(24-x)^{\alpha}(\alpha-2 \alpha c-1)$ is positive for $c>\frac{\alpha-1}{2 \alpha}$, and $\frac{\alpha-1}{2 \alpha}<\frac{\alpha-2}{2 \alpha}$ when $\alpha<-2$, the conclusion of the lemma follows. 
Claim 1: In region $A$, with $\theta>\theta(x, \alpha)$, there is a unique value $c^{*} \in(0,1)$ where $\tilde{U}_{x}(c)$ takes on its maximum value.

Proof: As $c \rightarrow 0, \tilde{U}_{x}^{\prime}(c) \rightarrow \infty$, so the value of $c$ that maximizes $\tilde{U}_{x}(c)$ is in the interval $(0,1]$. As $c \rightarrow 1$, the first term in equation (A.3.2) approaches $\infty$, the second term is finite, and the last term has the finite limit $g(x) \equiv-(24-x)^{\alpha}-\theta x^{\alpha}$. Whether $\tilde{U}_{x}^{\prime}(c)$ approaches $+\infty, 0$, or $-\infty$ as $c \rightarrow 1$ therefore depends on the sign of the last term, which is $f_{x}(c)$.

Since $\lim _{c \rightarrow 0} f_{x}(c)=\infty$ and $\lim _{c \rightarrow 1} f_{x}(c)=g(x)$ is negative for $\theta>\theta(x, \alpha), f_{x}(c)$ changes sign on $(0,1)$ at least once. By Lemma $1, f_{x}(c)$ changes sign at most twice in $(0,1)$ for $\alpha \in[-2,0) \cup(0,1)$ (and hence $\tilde{U}_{x}^{\prime}(c)$ changes sign at most twice). By Lemma $2, f_{x}(c)$ changes sign at most twice in $(0,1)$ for $\alpha \in(-\infty,-2)$. Therefore there are at most two roots of $f_{x}(c)=0$ in $(0,1)$ (and equivalently, there are at most two roots of the first order condition $\left.\tilde{U}_{x}^{\prime}(c)=0\right)$. As $c \rightarrow 1, f_{x}(c) \rightarrow g(x)$ and $g(x)<0$ in region $A$. Since $f_{x}(c)$ approaches a negative limit as $c \rightarrow 1$, it has a unique root in $(0,1)$, which demonstrates that $\tilde{U}_{x}^{\prime}(c)=0$ has a unique root in $(0,1)$.

Claim 2: In region $B_{1}$, with $\theta \leq \theta(x, \alpha)$ and $\alpha \in(0,1)$, we show that $\tilde{U}_{x}^{\prime}(c)>0$ for all $c \in(0,1)$, so that there is a boundary maximum of $\tilde{U}_{x}(c)$ at $c=1$, i.e., $q^{*}=24-x$.

Proof: The sign of $\tilde{U}_{x}^{\prime}(c)$ is the same as the sign of $f_{x}(c)$, so it is sufficient to show that $f_{x}(c)>0$ at its minimum on $(0,1)$. The argument below demonstrates first that $f_{x}(c)$ is decreasing on $(0,1)$ so that it takes on its minimum at $c=1$ and then shows that $f_{x}(1)>0$ so that $\tilde{U}_{x}^{\prime}(c)>0$ for all $c \in(0,1)$.

Since $f_{x}^{\prime}(c)=c^{\alpha-2}(24-x)^{\alpha}(c-2 c \alpha-1), f_{x}^{\prime}(c)<0$ if and only if $c-2 c \alpha-1<0$. The last inequality holds because $c<1$ so that $c-1<0$ and $\alpha c>0$ so that $-2 c \alpha<0$ for $\alpha<0$. Since this inequality holds for all $\alpha \in(0,1), f_{x}(c)$ is decreasing on $(0,1)$. Since $f_{x}(1)=g(x)$, and $g(x)$ is positive in region $B_{1}$, it follows that $\tilde{U}_{x}^{\prime}(c)>0$ for all $c \in(0,1)$.

Claim 3: In region $B_{2}$, with $\theta \leq \theta(x, \alpha)$ and $\alpha<0$, we show that for any $c^{\prime}<1, \tilde{U}_{x}(c)$ is bounded for $c \in\left[0, c^{\prime}\right]$ and $\tilde{U}_{x}(c) \rightarrow \infty$ as $c \rightarrow 1$ so that there is an asymptote of the utility function at $c=1$. Consequently, there is a boundary maximum of $\tilde{U}_{x}(c)$ at $c=1$, i.e., $q^{*}=24-x$.

Proof: It is clear from equation (A.3.1) that $\tilde{U}_{x}(c)$ is bounded for $c \in\left[0, c^{\prime}\right]$. As $c \rightarrow 1$, the term $(1-c)^{\alpha} \rightarrow \infty$ for $\alpha<0$, and the first and third terms are both finite, so $\tilde{U}_{x}(c) \rightarrow \infty$ if the last term, $c^{\alpha}(24-x)^{\alpha}+\theta x^{\alpha}$, tends to a negative limit as $c \rightarrow 1$. Since $\theta<\theta(x, \alpha)$ in region $B_{2}$ and this expression is equivalent to $(24-x)^{\alpha}+\theta x^{\alpha}<0$, the claim follows. 
Claim 4: For $\alpha=0$, there is a unique maximum of $U(x, q)$ at $q^{*}=\frac{24-x}{2+\theta}$ when $\theta>-1$ and there is a unique maximum of $U(x, q)$ at $q^{*}=24-x$ when $\theta \leq-1$.

Proof: This follows immediately from the utility maximization problem for $\alpha=0$.

Claim 5: For $\alpha=1$, there is a unique maximum of $U(x, q)$ at $q^{*}=12-\frac{1+\theta}{2} x$ when $\theta>\theta(x, 1)$ and there is a unique maximum of $U(x, q)$ at $q^{*}=24-x$ when $\theta \leq \theta(x, 1)$.

Proof: This follows immediately from the utility maximization problem for $\alpha=1$.

\section{Appendix A.4: Calculation of the Stackelberg best-response function $q^{*}(x ; \theta, \alpha)$}

Claims 2 and 3 in appendix A.3 demonstrates that for all $(x, \theta, \alpha) \in B_{1} \cup B_{2}$ (where $\theta<\theta(x, \alpha)$ ), $U(x, q)$ takes on its maximum at $q=24-x$. Claim 1 demonstrates that $(1)$ if $(x, \theta, \alpha) \in A$, then $U^{\prime}(x, 0)=\infty$ and $U^{\prime}(x, 24-x)<0$ and $(2) U^{\prime}(x, q)$ has a single root in $(0,24-x)$. We use (1) and (2) to calculate $q^{*}\left(x ; a, x_{0}, \alpha\right)$. Since the derivative is infinite at $q=0$, we start by evaluating $U^{\prime}(x, 1)$. If $U^{\prime}(x, 1)>0$ we use the secant method with $U^{\prime}(x, 1)$ and $U^{\prime}(x, 24-x)$ to find $q^{*}$ such that $U^{\prime}\left(x, q^{*}\right)=0$. If $U^{\prime}(x, 1)<0$, we bisect the interval until we find $2^{-k}$ such that $U^{\prime}\left(x, 2^{-k}\right)>0$, and then apply the secant method to identify $q^{*}$ such that $U^{\prime}\left(x, q^{*}\right)=0$.

\section{Appendix A.5: Stackelberg duopoly likelihood function}

As in the dictator game, for fixed values of the utility function parameters, the optimal choice is a function of $\epsilon$. Threshold values of $\epsilon$ determine the points at which optimal choices change. Suppose that $\epsilon$ has the error function distribution in equation (2). We want to determine threshold values $\epsilon_{q}$ such that $U\left(x, q ; \alpha, a, \theta\left(x, \epsilon_{q}\right), b, c\right)=U\left(x, q+1 ; \alpha, a, \theta\left(x, \epsilon_{q}\right), b, c\right)$, for $q \in\{3,4,5, \ldots, 15\}$. For $\epsilon \in\left(\epsilon_{3}, \infty\right)$, the optimal choice is $q=3$. For each $q \in\{4,5,6, \ldots, 14\}$, when $\epsilon \in\left(\epsilon_{q}, \epsilon_{q-1}\right)$, the optimal choice is $q$. When $\epsilon \in\left(-\infty, \epsilon_{14}\right)$ the optimal choice is $q=15$. The probability of $q$ can therefore be determined from the cumulative distribution function $F(z ; b, c)$ of the error function distribution.

The probability of $q=3$ is $1-F\left(\epsilon_{3} ; b, c\right)$. For $q \in\{4,5,6, \ldots, 14\}$ the probability of $q$ is $F\left(\epsilon_{q-1}, b, c\right)-F\left(\epsilon_{q} ; b, c\right)$. Finally, for $q=15$, the probability of $q$ is $F\left(\epsilon_{14} ; b, c\right)$. These probabilities determine the likelihood function

$$
L\left(q ; x, \alpha, a, x_{0}, \theta, b, c\right)=\prod_{i=1}^{220} \operatorname{Pr}\left[q_{i}=q \mid x_{i}, \alpha, a, x_{0}, \theta\left(x_{i}, \epsilon_{q}\right), b, c\right]
$$

and maximum likelihood estimates are obtained by maximizing this function (or its logarithm).

Standard errors estimates are obtained from the numerical Hessian matrix, as the square roots of its diagonal elements. 


\section{References}

[1] Abbink, Klaus, Bernd Irlenbusch, and Elke Renner (2000). "The Moonlighting Game: An Empirical Study on Reciprocity and Retribution," Journal of Economic Behavior and Organization 42, 265-77.

[2] Andreoni, James and John H. Miller (2002). "Giving According to GARP: An Experimental Test of the Consistency of Preferences for Altruism," Econometrica 70, 737-53.

[3] Berg, Joyce, John Dickhaut, and Kevin McCabe (1995). "Trust, Reciprocity, and Social History," Games and Economic Behavior 10, 122-42.

[4] Blount, Sally (1995). "When Social Outcomes Aren't Fair: The Effect of Causal Attributions on Preferences," Organizational Behavior and Human Decision Processes 63, 131-44.

[5] Bolton, Gary, Jordi Brandts, and Axel Ockenfels (1998). "Measuring Motivations for the Reciprocal Responses Observed in a Simple Dilemma Game," Experimental Economics 1, 207-19.

[6] Bolton, Gary E., Elena Katok, and Rami Zwick (1998). "Dictator Game Giving: Rules of Fairness versus Acts of Kindness," International Journal of Game Theory 27, 269-99.

[7] Bolton, Gary E. and Axel Ockenfels (2000). "ERC: A Theory of Equity, Reciprocity and Competition," American Economic Review 90, 166-93.

[8] Bolton, Gary E. and Rami Zwick (1995). "Anonymity versus Punishment in Ultimatum Bargaining," Games and Economic Behavior 10, 95-121.

[9] Bosman, Ronald and Frans van Winden (2002). "Emotional Hazard in a Power-to-Take Experiment," Economic Journal 112, 147-69.

[10] Brandts, Jordi and Gary Charness (2000). "Retribution in a Cheap Talk Experiment," UPF Barcelona manuscript.

[11] Cason, Timothy and Daniel Friedman (2003). "Buyer Search and Price Dispersion: A Laboratory Study.," Journal of Economic Theory 112, 232-60.

[12] Charness, Gary and Matthew Rabin (2002). "Understanding Social Preferences with Simple Tests," Quarterly Journal of Economics 117, 817-69.

[13] Cherry, Todd L., Peter Frykblom, and Jason Shogren (2002). "Hardnose the Dictator," American Economic Review, 92, 1218-21.

[14] Cox, James C. (2002). "Trust, Reciprocity, and Other-Regarding Preferences: Groups vs. Individuals and Males vs. Females," Experimental Business Research, Rami Zwick and Amnon Rapoport (eds.), Kluwer Academic Publishers. 
[15] Cox, James C. (2004). "How to Identify Trust and Reciprocity," Games and Economic Behavior 46, 260-81.

[16] Cox, James C. and Cary A. Deck (2005). "On the Nature of Reciprocal Motives," Economic Inquiry 43, 623-35.

[17] Cox, James C., R. Mark Isaac, Paula-Ann Cech, and David Conn (1996). "Moral Hazard and Adverse Selection in Procurement Contracting," Games and Economic Behavior 17, 147-76.

[18] Cox, James C. and Ronald L. Oaxaca (1989). "Laboratory Experiments with a Finite Horizon Job Search Model," Journal of Risk and Uncertainty 2, 301-29.

[19] Cox, James C. and Ronald L. Oaxaca (1996). "Is Bidding Behavior Consistent with Bidding Theory for Private Value Auctions?" Research in Experimental Economics, Vol. 6, R. Mark Isaac (ed.), Greenwich: JAI Press.

[20] Cox, James C. and Ronald L. Oaxaca (2000). "Good News and Bad News: Search from Unknown Wage Offer Distributions," Experimental Economics 2, 197-225.

[21] Cox, James C. and Vjollca Sadiraj (2005). "Direct Tests of Models of Social Preferences and Introduction of a New Model," University of Arizona working paper.

[22] Cox, James C., Klarita Sadiraj, and Vjollca Sadiraj (2004). "Implications of Trust, Fear, Reciprocity, and Altruism for Modeling Economic Behavior," University of Arizona working paper.

[23] Davidson, Russell, and James G. MacKinnon (1993). Estimation and Inference in Econometrics. Oxford University Press, Oxford U.K.

[24] Dufwenberg, Martin and Georg Kirchsteiger (2004). "A Theory of Sequential Reciprocity." Games and Economic Behavior 47, 268-98.

[25] Engelmann, Dirk and Martin Strobel (2004). "Inequality Aversion, Efficiency, and Maximin Preferences in Simple Distribution Experiments," American Economic Review 94, 857-869.

[26] Evans, Merran, Nicholas Hastings, and Brian Peacock (2000). Statistical Distributions, Third Edition, Wiley-Interscience, New York.

[27] Falk, Armin, Ernst Fehr, and Urs Fischbacher, (2003). "On the Nature of Fair Behavior," Economic Inquiry 41, 20-26.

[28] Falk, Armin and Urs Fischbacher (2006). "A Theory of Reciprocity," Games and Economic Behavior, 54, 293-315.

[29] Fehr, Ernst and Simon Gächter (2000a). "Cooperation and Punishment in Public Goods Experiments," American Economic Review 90, 980-94. 
[30] Fehr, Ernst and Simon Gächter (2000b). "Fairness and Retaliation: The Economics of Reciprocity," Journal of Economic Perspectives 14, 159-81.

[31] Fehr, Ernst, Simon Gächter, and Georg Kirchsteiger (1997). "Reciprocity as a Contract Enforcement Device: Experimental Evidence," Econometrica 65, 833-60.

[32] Fehr, Ernst and Klaus M. Schmidt (1999). "A Theory of Fairness, Competition, and Cooperation," Quarterly Journal of Economics 114, 817-68.

[33] Fisman, Raymond, Sachar Kariv and Daniel Markovits (2005). "Individual Preferences for Giving," Working paper, University of California, Berkeley.

[34] Gächter, Simon and Arno Riedl (2005). "Moral Property Rights in Bargaining with Infeasible Claims," Management Science, 51, 2, 249-263.

[35] Gale, John, Kenneth G. Binmore and Larry Samuelson (1995). "Learning to Be Imperfect: The Ultimatum Game," Games and Economic Behavior 8, 56-90.

[36] Geanakopolos, John, David Pearce, and Ennio Stacchetti (1989). "Psychological Games and Sequential Rationality," Games and Economic Behavior 1, 60-79.

[37] Güth, Werner, Rolf Schmittberger, and Bernd Schwarze (1982). "An Experimental Analysis of Ultimatum Bargaining," Journal of Economic Behavior and Organization 3, 367-88.

[38] Guttman, Joel M. (2000). "On the Evolutionary Stability of Preferences for Reciprocity," European Journal of Political Economy 16, 31-50.

[39] Harrison, Glenn W. and Peter Morgan (1990). "Search Intensity in Experiments," Economic Journal 100, 478-86.

[40] Hoffman, Elizabeth, Kevin McCabe, Keith Shachat, and Vernon L. Smith (1994). "Preferences, Property Rights, and Anonymity in Bargaining Games," Games and Economic Behavior 7, $346-80$

[41] Huck, Steffen, Wieland Müller, and Hans-Theo Normann (2001). "Stackelberg beats Cournot: On collusion and efficiency in experimental markets," Economic Journal 111, 749-766.

[42] Kagel, John H. and Katherine Wolfe (2001). "Tests of Fairness Models Based on Equity Considerations in a Three Person Ultimatum Game," Experimental Economics 4, 203-220.

[43] Konow, James (2000). "Fair Shares: Accountability and Cognitive Dissonance in Allocation Decisions," American Economic Review 90, 1072-1091.

[44] Konow, James (2001). "Fair and Square: The Four Sides of Distributive Justice," Journal of Economic Behavior and Organization 46, 137-164. 
[45] Konow, James (2003). "Which Is the Fairest One of All? A Positive Analysis of Justice Theories," Journal of Economic Literature 61, 1188-1239.

[46] Levine, David K. (1998). "Modeling Altruism and Spitefulness in Experiments," Review of Economic Dynamics 1, 593-622.

[47] Offerman, Theo (2002). "Hurting Hurts More than Helping Helps," European Economic Review 46, 1423-37.

[48] Rabin, Matthew (1993). "Incorporating Fairness into Game Theory and Economics," American Economic Review 83, 1281-1302.

[49] Slonim, Robert and Alvin E. Roth (1998). "Learning in High Stakes Ultimatum Games: An Experiment in the Slovak Republic," Econometrica 66, 569-96.

[50] Smith, Adam (1759). The Theory of Moral Sentiments. Reprinted by Indianapolis: Liberty Classics, 1976.

[51] Smith, Vernon L. and Arlington W. Williams (1990). "The Boundaries of Competitive Price Theory: Convergence, Expectations, and Transaction Costs," Advances in Behavioral Economics, vol. 2, L. Green and J.H. Kagel, eds., Norwood, NJ: Ablex Publishing Corp.

[52] Sobel, Joel (2005). "Interdependent Preferences and Reciprocity," Journal of Economic Literature XLIII, 392-436. 